\title{
Growth in Baumslag-Solitar groups I: subgroups and rationality
}

\author{
Eric M. Freden, Teresa Knudson and Jennifer Schofield
}

\begin{abstract}
The computation of growth series for the higher Baumslag-Solitar groups is an open problem first posed by de la Harpe and Grigorchuk. We study the growth of the horocyclic subgroup as the key to the overall growth of these Baumslag-Solitar groups $\mathrm{BS}(p, q)$, where $1<p<q$. In fact, the overall growth series can be represented as a modified convolution product with one of the factors being based on the series for the horocyclic subgroup. We exhibit two distinct algorithms that compute the growth of the horocyclic subgroup and discuss the time and space complexity of these algorithms. We show that when $p$ divides $q$, the horocyclic subgroup has a geodesic combing whose words form a context-free (in fact, one-counter) language. A theorem of Chomsky-Schützenberger allows us to compute the growth series for this subgroup, which is rational. When $p$ does not divide $q$, we show that no geodesic combing for the horocyclic subgroup forms a context-free language, although there is a context-sensitive geodesic combing. We exhibit a specific linearly bounded Turing machine that accepts this language (with quadratic time complexity) in the case of $\mathrm{BS}(2,3)$ and outline the Turing machine construction in the general case.
\end{abstract}

\section{Introduction and review of growth series}

Since their exhibition by Baumslag and Solitar [2], the family of groups named after them have been a frequent source of examples and counterexamples in group theory. The purpose of this article is to shed light on an open problem originally proposed by de la Harpe and Grigorchuk (see [7] for example), namely, compute the growth series for the groups $\operatorname{BS}(p, q)$ using the standard presentation $\left\langle b, t \mid t b^{p} t^{-1}=b^{q}\right\rangle$. When $p=1$ or when $p=q$, the growth series have been computed (see $[\mathbf{3}, \mathbf{6}, \mathbf{9}])$. However, when $1<p<q$, the growth computation appears to be a difficult problem. In fact, effective algorithms for finding geodesic representatives for group elements have only recently appeared in the solvable case [10], and more generally whenever $p$ divides $q[\mathbf{8}]$. The latter article also exhibits a proof of our Corollary 9.2 below using different methods.

We concentrate our efforts on the relative growth of the cyclic subgroup generated by $b$. The motivation for this strategy is that $\mathrm{BS}(p, q)$ is a disjoint union of cosets of this subgroup, and the growth on each coset is related to the growth of the subgroup. Rather than attempt to find and count normal forms for each element of $\mathrm{BS}(p, q)$, we try to write the growth series of the group as a modified convolution based on relative growth of each coset (cf. Sections 2 and 3 below).

There are at least two sources of complexity associated to the growth of $\operatorname{BS}(p, q)$. Geometry in the form of gluing negatively curved half-sheets along positively curved horocycles is the first issue (see Section 2 below for definitions). The second issue is number theoretic. Only the latter is problematic in this article. Indeed, the geometry examined in this article is restricted to negative curvature and is a useful tool (cf. Proposition 8.1). In the following [14], where we study growth exponents, the alternation of positive and negative curvatures presents a formidable obstacle. Number-theoretic issues arise in Sections 6 and 7 below.

We begin with a short review of generating functions. Let $B(z)=\sum_{r=0}^{\infty} \beta_{r} z^{r}$ be a power series. Recall that the radius of convergence is defined as the reciprocal of

$$
\omega=\limsup _{r \rightarrow \infty}\left(\beta_{r}\right)^{1 / r}
$$

Received 29 June 2009; revised 12 May 2010.

2010 Mathematics Subject Classification 20E07 (primary), 20F65, 20F10, 68Q45 (secondary). 
Let $G=\langle\mathcal{X} \mid \mathcal{R}\rangle$ be a presentation for a finitely generated group and let $\beta_{r}$ (respectively $\sigma_{r}$ ) be the number of elements $g$ in $G$ whose word length is at most (respectively equal to) $r$. Then recall that the above sum $B(z)$ (or $S(z)=\sum_{r=0}^{\infty} \sigma_{r} z^{r}$, respectively) is called the cumulative (respectively spherical) growth series for the presentation. For either growth series, the above limit superior is actually an ordinary limit [7], and is called the exponent of growth. This $\omega$ is a number in $[1,+\infty)$ and is the reciprocal of the modulus of the smallest singularity for $B(z)$. Recall that the formal convolution product

$$
\sum_{r=0}^{\infty} c_{r} z^{r}=\left(\sum a_{r} z^{r}\right)\left(\sum_{r=0}^{\infty} b_{r} z^{r}\right)
$$

has coefficients defined by $c_{r}=\sum_{j=0}^{r} a_{j} b_{r-j}$. By definition, $\beta_{r}=\sum_{j=0}^{r} \sigma_{j} \cdot 1$. We see that the cumulative growth series $B(z)$ is the convolution product of the spherical growth series $S(z)$ with the geometric series $\sum_{r=0}^{\infty} z^{r}=\frac{1}{1-z}$. Therefore, $B(z)=\frac{S(z)}{1-z}$. The smallest singularity of any meromorphic function $B(z)$ is completely determined by $S(z)$, since $\frac{1}{1-z}$ contributes a singularity of 1 . This is the largest radius of convergence possible since its reciprocal must lie in $[1,+\infty)$. Thus, both $S(z)$ and $B(z)$ have the same radius of convergence, the same exponent of growth $\omega$, and each is a rational function if and only if the other is a rational function.

It is well known that $S(z)=\sum_{r=0}^{\infty} \sigma_{r} z^{r}$ is a rational function (that is, a quotient of two polynomials) if and only if the coefficients satisfy a linear, recurrence relation with constant coefficients for all sufficiently large $r$. In this case, the denominator of $S(z)$ is completely determined by the recurrence, that is,

$$
\begin{aligned}
& \sigma_{r}=k_{1} \sigma_{r-1}+k_{2} \sigma_{r-2}+\ldots+k_{j} \sigma_{r-j}
\end{aligned}
$$

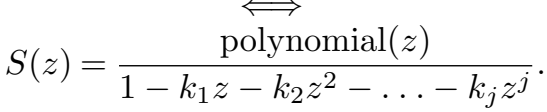

The coefficients of the numerator polynomial are determined by the values of the initial terms $\sigma_{0}, \sigma_{1}, \ldots$ prior to the recursion. The exponent of growth for rational $S(z)$ with integer coefficients is necessarily an algebraic number.

The remainder of the article is outlined as follows. Section 2 reviews the coarse geometry of the Cayley 2-complex for a Baumslag-Solitar group. Section 3 is a brief sketch of some of our methodology to recompute the growth function for $\mathrm{BS}(1,3)$, that was first found in [6]. Section 4 describes an algorithm discovered by Alisha McCann in 2002 to convert horocyclic paths into so-called McCann geodesics. Section 5 comprises a derivation of the growth series for the horocyclic subgroup of $\mathrm{BS}(2,4)$ via geometry and formal language theory. Section 6 studies the horocyclic subgroup of $\mathrm{BS}(2,3)$, defines another set of the normal forms (the Schofield geodesics), and constructs a Turing machine to accept these forms along with time and space complexity estimates. Section 7 shows that when $p \nmid q$, no geodesic combing of the horocyclic subgroup can constitute a context-free language. Section 8 generalizes Section 6 to any $\operatorname{BS}(p, q)$, where $p \nmid q$. Section 9 modifies the Turing machine of earlier sections into a one-counter machine in the case when $p$ divides $q$, and shows that the corresponding horocyclic subgroup has rational growth. The appendix to this article lists context-free grammars that describe geodesic combings for the horocyclic subgroup in all cases where $p \mid q$ (these grammars are a compact method of encoding growth functions).

\section{The geometry of Baumslag-Solitar groups}

For the general group $\mathrm{BS}(p, q)$ in this genre, we call $\langle b\rangle$ the horocyclic subgroup and the defining relator $t b^{p} t^{-1} b^{-q}$ is referred to as a 'horobrick' in [12]. The Cayley graph consists of 'sheets' each of which is endowed with a coarse euclidean geometry (when $p=q$ ) or coarse hyperbolic 


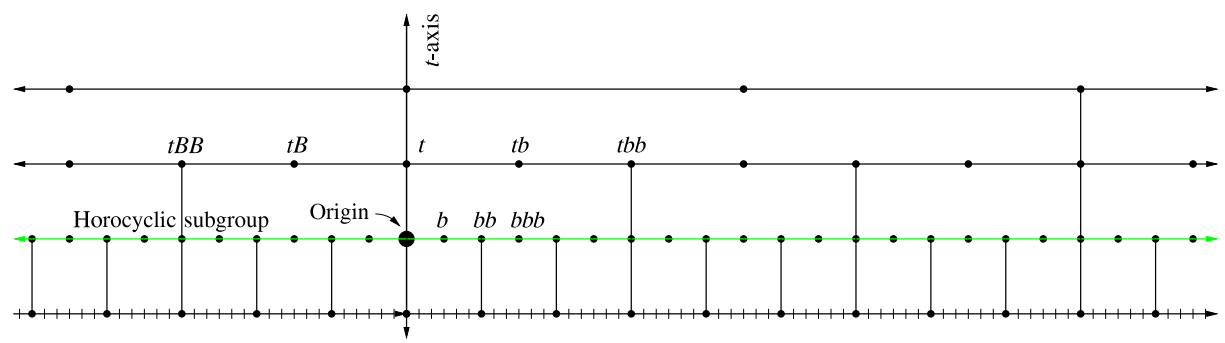

Figure 2.1. Partial sheet for BS(2,6) with horocyclic subgroup.

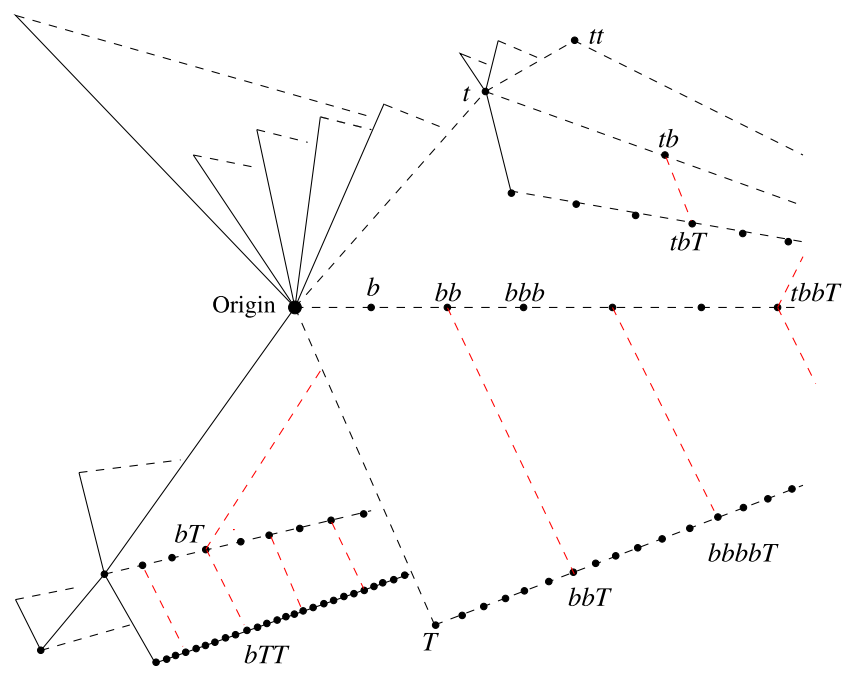

Figure 2.2. Cut-away view of Cayley 2-complex of BS(2,6); solid lines represent cut lines, actual generator edges are dashed. Each horocycle should have six upward and two downward branch sheets (most are omitted from the figure for brevity).

geometry (when $p<q$ ) glued along $\langle b\rangle$-cosets referred to as 'horocycles'. This geometry is quasi-isometric with the upper half space model of the hyperbolic plane (and thus satisfies the thin-triangle criterion, making each sheet a Gromov hyperbolic space). The quasi-isometry induces a natural orientation to each sheet. Paths with labels $t^{n}, n>0$, go 'up', labels $b^{ \pm n}$ are 'horizontal', while paths labeled $t^{-n}$ go 'down' (see Figure 2.1).

Recall that the Cayley 2-complex of a presentation is obtained from the Cayley graph by filling in each basic relator and its conjugates with a topological disk. For $\mathrm{BS}(p, q)$, the Cayley 2-complex is homeomorphic to the product of the real line with a simplicial tree (see Figure 2.2). This tree is in fact the Bass-Serre tree upon which the group acts.

Consider any strictly upward line (or ray) in the Bass-Serre tree. By a sheet (or half-sheet), we mean the full lift of this path in the Cayley graph. We will often use a restricted version of a so-called upper half-sheet: we require each such half-sheet to contain some half-coset $\left\{w t^{n}: n>0\right\}$, where $w$ is a geodesic word with suffix $b$ or $b^{-1}$. We think of $w$ as a connecting segment from the origin (identity vertex) to the half-sheet. Similarly, each restricted lower halfsheet must contain a half-coset of the form $\left\{w t^{-n}: n>0\right\}$, where $w$ is a geodesic word with suffix $b$ or $b^{-1}$. There are only countably many half-sheets using these definitions. The geodesic word $w$ above is termed a stem associated to the (restricted version of the) half-sheet. The notation $|w|$ refers to the minimal word length of any word $v$ representing the same element as $w$ in the group $\mathrm{BS}(p, q)$. By this definition, $w$ is a geodesic if and only if the word length of $w$ 


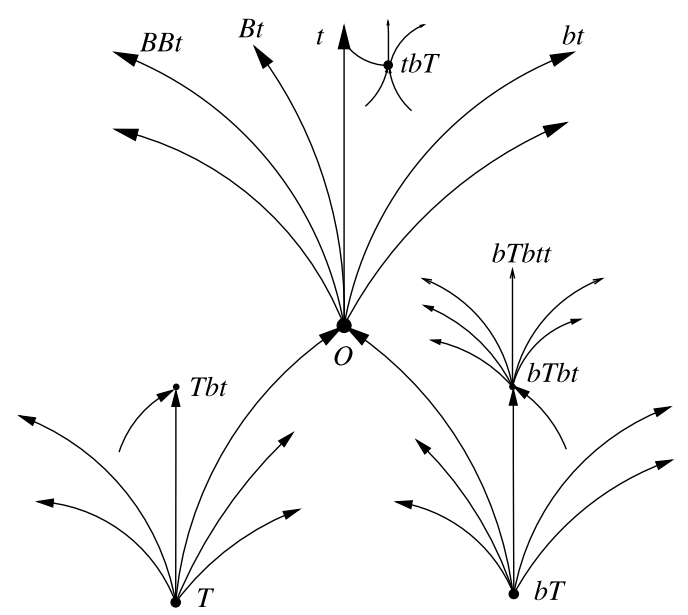

Figure 2.3. Partial Bass-Serre tree for $\mathrm{BS}(2,6)$.

(in the free group on $b, t$ ) equals $|w|$. We frequently abuse notation and allow $w$ to also refer to the ending vertex of the edge path starting from the origin that is labeled by the word $w$. In this context, $|w|$ means the metric distance between the origin and the vertex $w$. We also use the synonym radius of $w$ to denote this distance.

Since the defining relator must be maintained in each sheet, each $\langle b\rangle$-coset of $\operatorname{BS}(p, q)$ gives rise to $q$ upper half-sheets and $p$ lower half-sheets. If Figure 2.2 is rotated so that the horocycles extend along the reader's line of sight, the Cayley graph projects to the simplicial tree mentioned earlier. For example, Figure 2.3 illustrates part of the tree for $\operatorname{BS}(2,6)$ with each dot representing a horocycle.

We frequently adopt monoid notation and denote $b^{-1}$ and $t^{-1}$ by $B$ and $T$, respectively. This is useful for representing strings in Perl and Java code and also for annotating graphics.

The family $\operatorname{BS}(q, q)$ has euclidean sheets joined along geodesic $\langle b\rangle$-cosets. The upper and lower half-sheets merge together into copies of the integer lattice (with some vertical edges missing). Each of these groups is automatic and has rational growth explicitly computed in [9]. The next simplest family are the solvable groups $\mathrm{BS}(1, q)$. These also have rational growth, as shown in $[3,6]$. The methodology used to prove these results is standard combinatorial group theory: find a geodesic normal form for each group element and count these forms. This idea has been unsuccessful so far when applied to $\operatorname{BS}(p, q)$, where $1<p<q$.

We propose the use of geometry to compute the growth series for the horocyclic subgroups $\langle b\rangle$ (as suggested by L. Bartholdi), as well as the entire growth series for certain groups. Even when a growth series cannot be exactly computed, our methods allow us to determine or estimate the exponents of growth (as suggested by A. Talambutsa). The latter strategy is based on the following key idea: estimate the growth of each horocycle and count the various horocycles. Counting the branching of horocycles is equivalent to counting the different geodesic stems $w$ used to define the horocycles. The growth on the horocycles we consider depends directly on the growth of the corresponding horocyclic subgroup $\langle b\rangle$. As a reality check, both aspects of our program can be applied to the automatic and solvable groups and compared with the already known growth functions.

\section{Motivation: the solvable case}

The subfamily $\mathrm{BS}(1, q)$ are solvable groups. The subgroup $\langle b\rangle$ is a horocycle that divides the main sheet into one lower and $q$ many upper half-sheets. The growth function (either spherical 


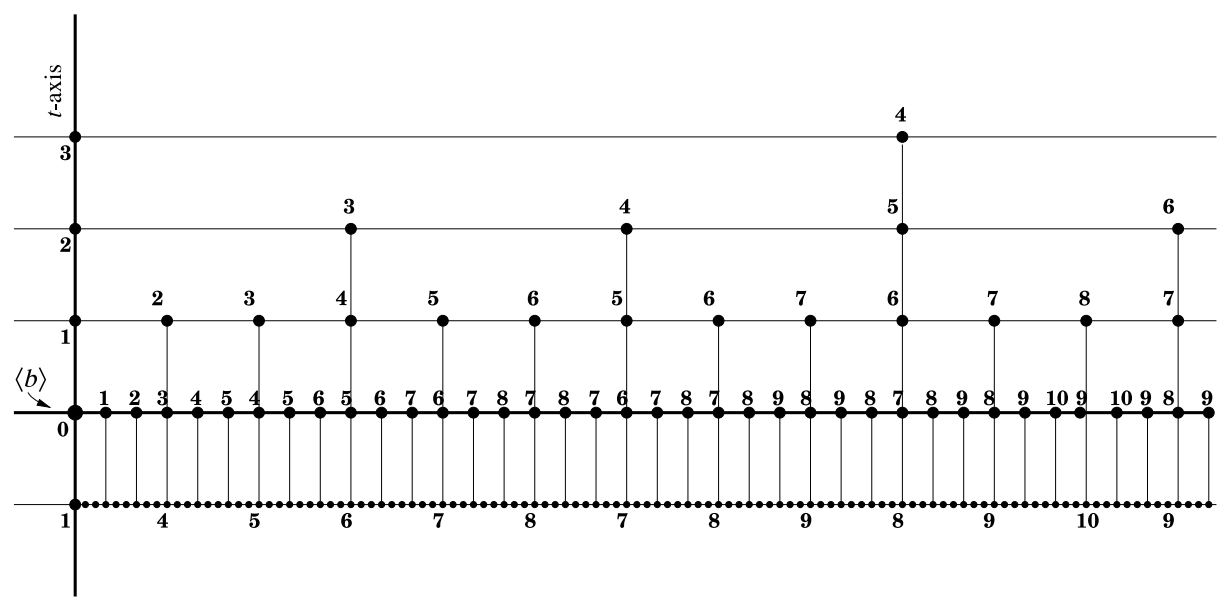

Figure 3.1. Part of the main sheet for $\mathrm{BS}(1,3)$ showing absolute distances from the origin.

or cumulative) on any of these grows exponentially with respect to radius. It is not difficult to see that the spherical growth of 'Quadrant I' on the upper main half-sheet (see Figure 3.1) is exactly the cumulative growth of half the horocycle $\left\{b^{n}: n>0\right\}$ (in fact, this holds for all the Baumslag-Solitar groups). This is because the cosets $t\langle b\rangle, t^{2}\langle b\rangle, t^{3}\langle b\rangle, \ldots$ comprising the main upper half-sheet have spherical growth counts that are exactly offset by $1,2,3, \ldots$ from that of $\langle b\rangle$.

The lower half-sheet consists of horocycles that eventually follow the growth pattern of $\langle b\rangle$, but different behavior is exhibited near the origin (we discuss this in terms of level in the next paragraph). The growth on the lower half-sheet always outpaces that of any upper half-sheet (consider a circle in the hyperbolic plane divided through its center by a horocycle - the convex piece of this disk has smaller area).

The growth function of any horocycle depends recursively on that of an adjacent horocycle. Here the notion of 'coset level' comes into play. Recall that the generator $t$ defines a vertical direction in the Cayley graph. We define the horocyclic subgroup to have level zero. Any horocycle adjacent to and immediately higher than a horocycle of level zero also has level zero. Any horocycle adjacent to and immediately lower than a horocycle of level $-n \leqslant 0$ is defined to have level $-n-1$. Any horocycle adjacent to and immediately higher than a horocycle of level $-n<0$ is defined to have level $-n+1$. Thus, the horocycles $t^{4} b t\langle b\rangle, b t^{-3} b t\langle b\rangle$ have levels 0 and -2 , respectively. It is clear that each level is merely an equivalence class of horocycles.

The first step in computing the overall growth function is to determine a recursion for the horocyclic subgroup and then find the growth recursion along any $\langle b\rangle$-coset as a function of level. We then count how many horocycles there are at a given level, form a convolution product with the recursion along such a coset, and finally sum over all levels.

As an example, consider the case of BS $(1,3)$. Define $b(0, r)$ to be the number of $b^{k}$ such that $\left|b^{k}\right|=r$. This defines the spherical growth series for the horocyclic subgroup, which satisfies

$$
b(0,0)=1, b(0,1)=b(0,2)=b(0,3)=2, b(0,4)=4
$$

and

$$
b(0, r)=b(0, r-2)+2 b(0, r-3)
$$

for all $r \geqslant 5$. The reader is invited to derive the recursion or borrow it from [6], where it is captured in the denominator of the growth series for $\mathrm{BS}(1,3)$. Multiplying both sides of the 
recursion by $z^{r}$ and summing over all $r \geqslant 5$ gives

$$
\sum_{r=5}^{\infty} b(0, r) z^{r}=z^{2} \sum_{r=5}^{\infty} b(0, r-2) z^{r-2}+2 z^{3} \sum_{r=5}^{\infty} b(0, r-3) z^{r-3} .
$$

Now use the definition $B_{0}(z)=\sum_{r=0}^{\infty} b(0, r) z^{r}$ and initial conditions to rewrite the equation above:

$$
B_{0}(z)-1-2 z-2 z^{2}-2 z^{3}-4 z^{4}=z^{2}\left(B_{0}(z)-1-2 z-2 z^{2}\right)+2 z^{3}\left(B_{0}(z)-1-2 z\right)
$$

and solve to get the generating function for the horocyclic subgroup

$$
B_{0}(z)=\frac{1+2 z+z^{2}-2 z^{3}-2 z^{4}}{1-z^{2}-2 z^{3}}=\frac{(1-z)(1+z)\left(1+2 z+2 z^{2}\right)}{1-z^{2}-2 z^{3}} .
$$

Given any other horocycle $K$ of level zero, there is a (unique) element $w$ of $K$ with minimal geodesic length such that $K=w\langle b\rangle$. Treat $w$ as a relative origin for $K$, in the sense that it has relative distance zero. Then it is true that $b(0, i)$ also counts the number of elements $g$ of $K$ satisfying $|g|-|w|=i$. A straightforward induction argument shows that the growth coefficients for any such level zero coset $K$ are the same as those of the horocyclic subgroup except for a shift of the indices by $|w|$ (the details can be found in [15]).

A relation between spherical counts on horocycles is $b(-n, i)=b(-n+1, i)+2 b(-n+1$, $i-1)$, where the arguments $-n$ and $-n+1$ refer to level and the other argument refers to the difference of distances as explained in the previous paragraph. (Again, the reader may derive this as an exercise or see [15, Section 1].) This recursion is valid for all $n, i \geqslant 1$. Again, multiply each side by $z^{i}$, sum over all $i \geqslant 1$, and use the initial condition $b(-n, 0)=1$ to derive

$$
B_{-n}(z)=(1+2 z) B_{-n+1}(z) \text {. }
$$

This equation is valid for all $n \geqslant 1$ and immediately implies

$$
B_{-n}(z)=(1+2 z)^{n} B_{0}(z)=\frac{(1-z)(1+z)\left(1+2 z+2 z^{2}\right)(1+2 z)^{n}}{1-z^{2}-2 z^{3}},
$$

which is valid for all $n \geqslant 0$.

Now define $\chi(-n, r)$ as the number of cosets at level $-n$ whose closest point projection to the identity element is $r$. In other words, $\chi(-n, r)$ counts all of the cosets $w\langle b\rangle$ with $|w|=r$. A glance at Figure 3.2 will confirm that $\chi(0,0)=1, \chi(0,1)=1, \chi(0,2)=3$ and, more generally, $\chi(-n, r)=0$ for all $0 \leqslant r<n$ and $\chi(-n, n)=1, \chi(-n, n+1)=0=\chi(-n, n+2), \chi(-n$, $n+3)=2$. Furthermore, Figure 3.2 illustrates the branching recursion

$$
\chi(-n, r)=\chi(-n+1, r-1) \text { for all } n>1 \text { and } r \geqslant n+3 .
$$

Define the coset generating functions $X_{-n}(z)=\sum_{r=0}^{\infty} \chi(-n, r) z^{r}$. Multiply each side of the recursion by $z^{r}$, for all $r \geqslant n+3$, and use the initial conditions to derive

$$
X_{-n}(z)=z X_{-n+1}(z)=z^{n-1} X_{-1}(z) \quad \text { valid for all } n \geqslant 1 .
$$

In order to determine $X_{-1}(z)$, Figure 3.2 again shows how horocycles propagate from a horocycle directly underneath:

$$
\chi(-n, r)=\chi(-n-1, r-1)+2 \chi(-n-1, r-2) \quad \text { for all } n>0 \text { and } r \geqslant n+3 .
$$

Put $n=1$ and use relation (3.3) to obtain

$$
\chi(-1, r)=\chi(-1, r-2)+2 \chi(-1, r-3) \text { for all } r \geqslant 4,
$$

which is the same recursion as that of the horocyclic subgroup, albeit with different initial conditions. The (by now) familiar method yields the generating function

$$
X_{-1}(z)=\frac{z(1-z)(1+z)}{1-z^{2}-2 z^{3}}
$$




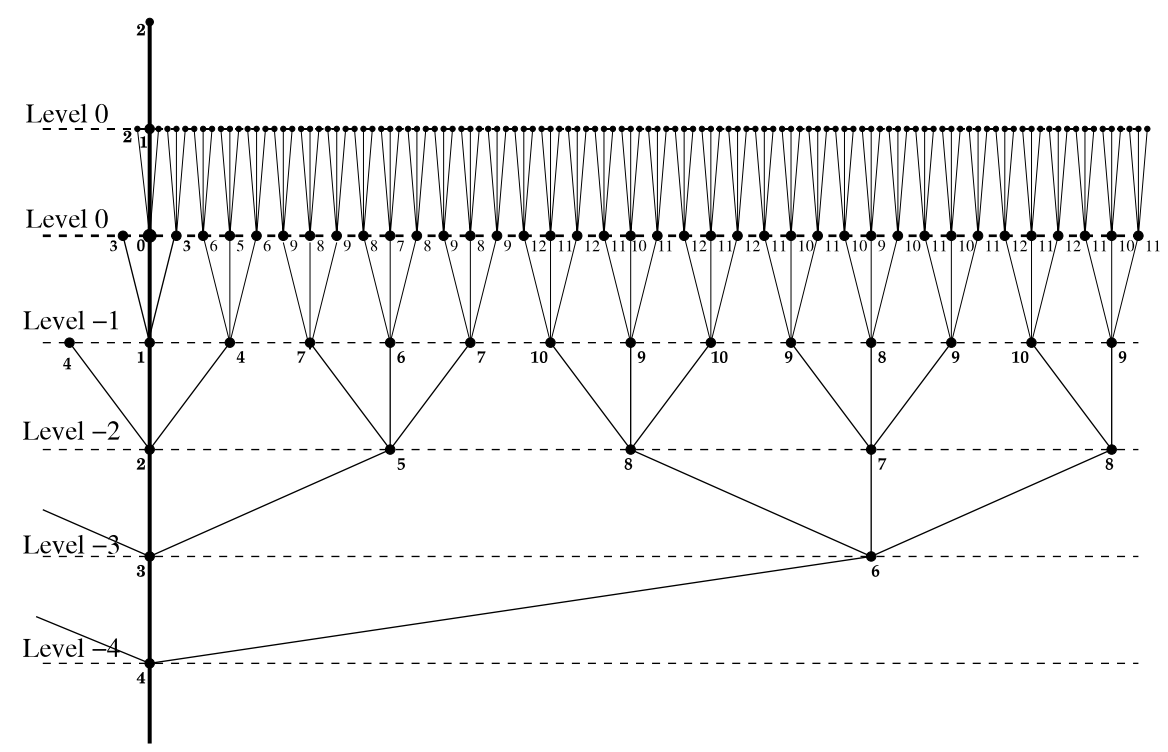

FiguRE 3.2. Branching for BS(1,3) illustrating absolute distances and levels.

and hence

$$
X_{-n}(z)=\frac{z^{n}(1-z)(1+z)}{1-z^{2}-2 z^{3}} \text { for all } n>0 .
$$

In order to find $X_{0}(z)$, we examine how new level-zero horocycles arise from already counted horocycles of level zero and from level minus one. In fact,

$$
\chi(0, r)=\chi(0, r-1)+2 \chi(0, r-2)+\chi(-1, r-1)+2 \chi(-1, r-2) \quad \text { for all } r \geqslant 3 .
$$

Substitute the branching recursion $\chi(-1, r-1)+2 \chi(-1, r-2)=\chi(-1, r+1)$ stated earlier and solve for

$$
X_{0}(z)=\frac{1}{z(1+z)(1-2 z)} X_{-1}(z)=\frac{1-z}{(1-2 z)\left(1-z^{2}-2 z^{3}\right)} .
$$

Define $\sigma(-n, r)$ as the number of vertices in the Cayley graph at level $-n$ whose radius from the identity is $r$. For fixed $n \geqslant 0$, we count $\sigma(-n, r)$ via the convolution

$$
\sigma(-n, r)=\sum_{i=0}^{r} \chi(-n, i) b(-n, r-i) .
$$

This is easily visualized: if $g$ is an element at level $-n$ and has geodesic length $r$, then $g$ lies on some horocycle $w\langle b\rangle$, where $w$ is a geodesic stem of length $i$. There is always a geodesic path for $g$ having prefix $w$. The number $b(-n, r-i)$ counts the number of elements on the horocycle $w\langle b\rangle$ at distance $r-i$ from $w$. There are exactly $\chi(-n, i)$ such cosets.

Let $S_{-n}(z)$ denote the generating function for this sequence. Combining (3.1) and (3.8), we see

$$
S_{0}(z)=X_{0}(z) B_{0}(z)=\frac{(1-z)^{2}(1+z)\left(1+2 z+2 z^{2}\right)}{(1-2 z)\left(1-z^{2}-2 z^{3}\right)^{2}}
$$

and, for $n>0$, combining (3.2) with (3.7) yields

$$
S_{-n}(z)=X_{-n}(z) B_{-n}(z)=\frac{(1-z)^{2}(1+z)^{2}\left(1+2 z+2 z^{2}\right) z^{n}(1+2 z)^{n}}{\left(1-z^{2}-2 z^{3}\right)^{2}} .
$$


The entire spherical count for the group is $\sigma_{r}=\sum_{n=0}^{r} \sigma(-n, r)$, which implies that the overall generating function is

$$
\begin{aligned}
S(z) & =\sum_{n=0}^{\infty} S_{-n}(z)=S_{0}(z)+\sum_{n=1}^{\infty} S_{-n}(z) \\
& =S_{0}(z)+\frac{(1-z)^{2}(1+z)^{2}\left(1+2 z+2 z^{2}\right)}{\left(1-z^{2}-2 z^{3}\right)^{2}} \sum_{n=1}^{\infty} z^{n}(1+2 z)^{n} \\
& =S_{0}(z)+\frac{(1-z)^{2}(1+z)^{2}\left(1+2 z+2 z^{2}\right)}{\left(1-z^{2}-2 z^{3}\right)^{2}}\left(\frac{1}{1-z(1+2 z)}-1\right) \\
& =\frac{(1-z)^{2}(1+z)\left(1+z+2 z^{2}\right)\left(1+2 z+2 z^{2}\right)}{(1-2 z)\left(1-z^{2}-2 z^{3}\right)^{2}} .
\end{aligned}
$$

This is in agreement with [6]. Note that the exponent of growth is 2 , coming from the $(1-2 z)$ factor in the denominator. This factor arises from the branching process of horocycles at level zero. We see that the exponent of growth depends only on the branching of the coset tree. Our method extends with only minor modification to $\mathrm{BS}(1, q)$, for any odd $q$. For even $q$, the branching of cosets is somewhat more involved, but this geometric/convolution method is still far simpler than the traditional means used in [6]. Another benefit of the geometric method is that the exponent of growth can be explicitly computed even when the horocyclic subgroup growth function cannot be explicitly computed. In fact, for $\mathrm{BS}(1, q)$ it is not difficult to show that $\omega \rightarrow 1+\sqrt{2}$ as $q \rightarrow \infty$ because the branching of the coset tree obeys a linear recursion. Finally, our method was successfully employed in [15] to compute a non-rational growth series.

\section{The horocyclic subgroup: common aspects}

As remarked above, the growth series for the horocyclic subgroup in either the solvable or automatic cases is rational and known. The remaining groups can be partitioned into two cases depending on whether $p$ divides $q$ or not. Before examining these cases, we discuss an algorithm written by Alisha McCann and revised by Jennifer Schofield that can (in principle) find the geodesic length of any word $b^{k}$ in any Baumslag-Solitar group. The original McCann reduction algorithm written in Perl accepts an input string of the form $b^{k} \in \mathrm{BS}(2,3)$ and outputs a corresponding geodesic representative string. In principle, this algorithm constructs a geodesic combing for the horocyclic subgroup which we denote as the language of McCann geodesics $\mathcal{M}$. The numeric generalization of the algorithm accepts as input $p, q$ and the exponent $k$ and returns the numeric geodesic length for $b^{k} \in \mathrm{BS}(p, q)$. We give a pseudo-code description of this idea in Figure 4.1. (The author Schofield has radically improved the execution of the algorithm at the expense of a more complicated description; the reader is invited to download this code at $[\mathbf{1 8}]$.)

The validity of the McCann algorithm is based on geometric ideas and is proved by induction. As basis step, $b^{1}$ through $b^{p}$ are already geodesic words. Now we make two observations: first, assuming that $w$ is a geodesic representation for $b^{d p}$, then $t w T$ is a geodesic representation for $b^{d q}=t b^{d p} T$ (and, conversely, if $t w T$ is geodesic for some $b^{k}$, then $k$ has factorization $d p$ and $w$ is geodesic for $b^{d p}$ ). Second, any (sufficiently large) power of $b$ has a geodesic representative lying on the main upper half-sheet with the form of a 'mesa' described below. With these ideas at hand, suppose that the geodesic length of each $b^{k} \in \mathrm{BS}(p, q), 1 \leqslant k<L$, has been computed.

Let us examine $b^{L}$. If $L$ is congruent to zero modulo $q$, then $b^{L}$ can be written as the conjugate $t b^{p L / q} T$. The geodesic length of $b^{p L / q}$ is already known by hypothesis and thus $\left|b^{L}\right|$ measures two units more. On the other hand, if $L$ is not congruent to zero $\bmod q$, we do two computations 


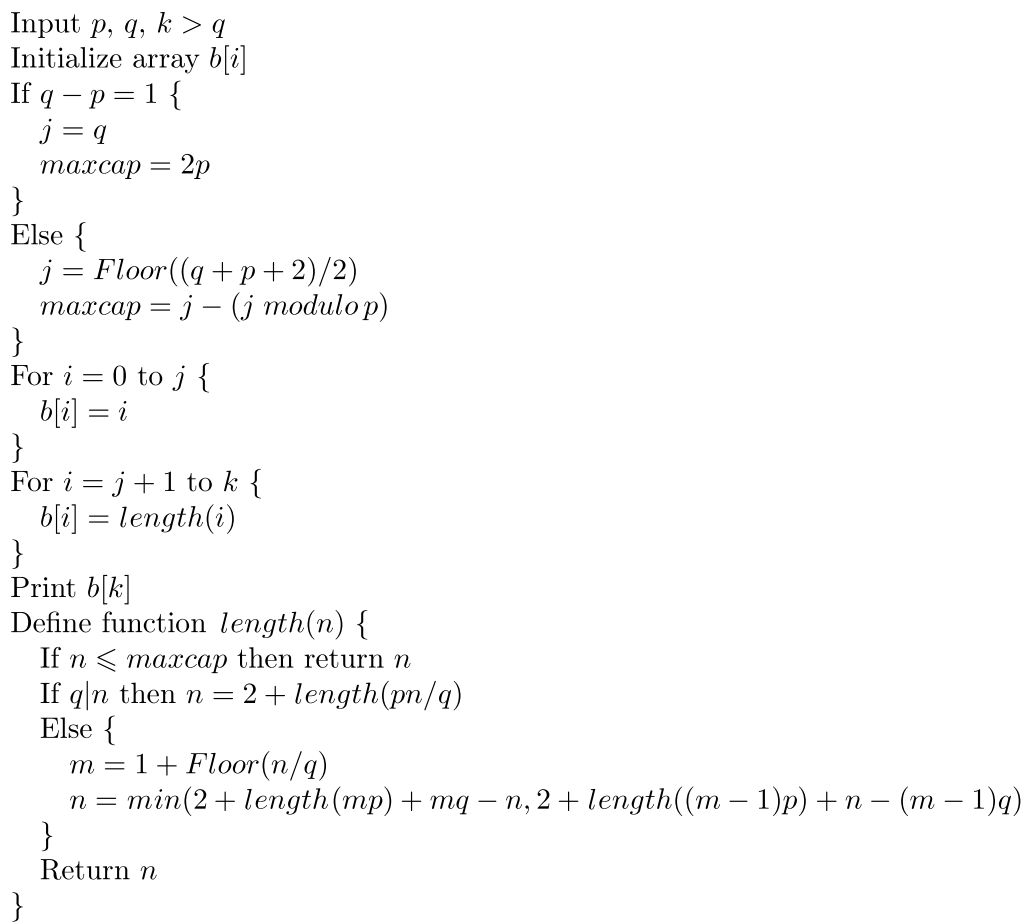

FIgURE 4.1. The McCann reduction algorithm.

and choose the smaller length: concatenate (delete, respectively) occurrences of $b$, in order to produce $b^{m q}$ (respectively $b^{(m-1) q}$ ), where $m q$ is the nearest multiple of $q$ that is larger than $L$. As noted earlier, we can rewrite $b^{m q}$ as $t b^{m p} T$, and the geodesic length of $b^{m p}$ is known by the induction hypothesis. We add two units to this length to account for the conjugation and then add $m q-L$ more units. Now perform the analogous length computation on $t b^{(m-1) p} T$ and add $L-(m-1) q$ units and compare with the sum of the previous sentence. The numerical McCann algorithm picks the smaller of these alternatives as the geodesic length for $b^{L}$. If both alternatives yield the same length, a choice is made (choose the first option, for example). If we restrict ourselves solely to the main upper half-sheet, the McCann algorithm produces shortest paths.

Perhaps there are even shorter paths when we relax the restriction about staying in the main upper half-sheet? First recall that the basic relation $t b^{p}=b^{q} T, 1<p<q$ implies that no geodesic for $b^{k}$ (large $k$ ) will go down, across, and back up around a horobrick. Furthermore, every geodesic for $b^{k}$ lies in some upper half-sheet (as per the definition of sheets in Section 2).

Let $L>0$ be the smallest integer for which $b^{L}$ has a representative word $w$ that does not lie on the main upper half-sheet and such that $w$ is strictly shorter than the McCann word $\gamma$ representing $b^{L}$. Note that $L$ is at least as large as the maximal cap length for the given $\operatorname{BS}(p, q)$. Since $w$ leaves the main sheet, it has the form $w=b^{u} t W T b^{v}$, where $u$ is the initial offset, $v$ is the terminal offset, and $W$ is a geodesic for the second stratum $s$ of $w$ (see Figure 4.2) immediately above $b^{L}$ in the new sheet. By the minimality hypothesis on $L$, we may assume that $W$ is in fact a McCann geodesic for $s$.

The supposed geodesic $w$ has an initial horizontal offset of $|u|>0$ units and a terminal horizontal offset of $|v| \geqslant 0$. Consider the conjugate $b^{-u} w b^{u}$, which can be freely reduced to the word $t W T b^{v} b^{u}$ which has the same word length as $w$. This word still represents $b^{L}$ but now resides completely in the main upper half-sheet, and even has the appearance of a McCann 


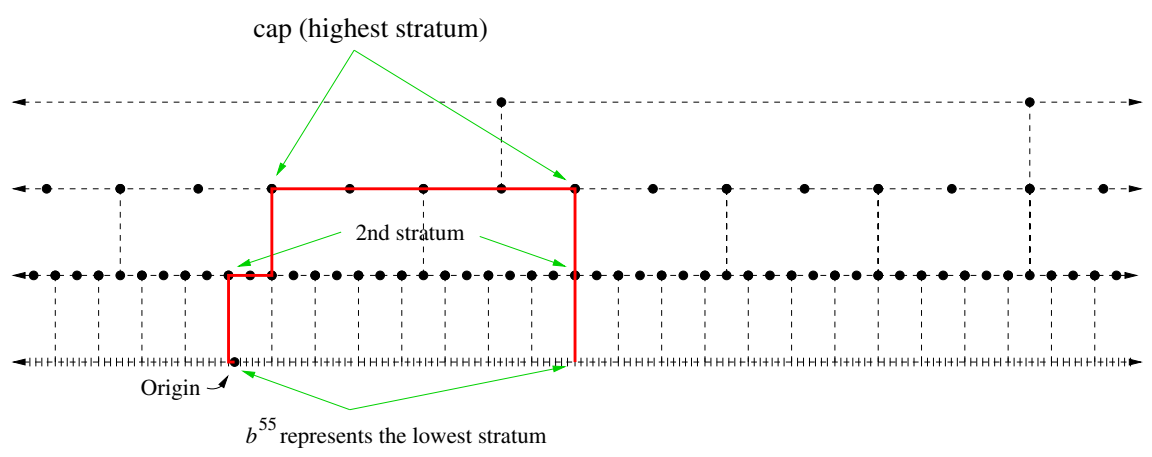

Figure 4.2. A Schofield geodesic in $\mathrm{BS}(2,7)$ and its strata.

path. However, the actual McCann path $\gamma$ for $b^{L}$ has minimal length (on the main upper half-sheet), as shown earlier. We obtain the inequalities

$$
|\gamma| \leqslant\left|t W T b^{v} b^{u}\right| \leqslant\left|t W T b^{v}\right|+|u|=|w|<|\gamma|,
$$

which comprise a contradiction. We conclude that no such $w$ is possible and we have proved the following proposition.

Proposition 4.1. The McCann algorithm calculates geodesic lengths for each $b^{k}, k>0$.

We note that the algorithm taking input $k$ creates an array of radii with $k$ indices in order to compute the geodesic length of $b^{k}$. It appears at first glance that the overall space usage is of linear magnitude. However, the input is not in unary and requires on the order of $\log k$ bits, while the array storage is on the order of $k \log k$ bits. Still, with several GB of RAM, Schofield's optimized version of the code runs very quickly [18].

As remarked earlier, the original McCann word reduction algorithm for $\mathrm{BS}(2,3)$ goes through the same sequence of steps but stores the actual string of generator letters in order to output a geodesic word representing $b^{L}$. We call the output word the McCann geodesic for $b^{L}$. The proof that the original McCann word reduction algorithm outputs geodesics is entirely analogous to the argument given above and is left to the interested reader.

The set of all McCann geodesics $\mathcal{M}$ have the following common aspects. Each member has a vertical prefix $t^{*}$ followed by a horizontal cap $b^{n}$, where $n$ depends on the values of $p$ and $q$; for example, we can take $n=4$ in the case of $\mathrm{BS}(2,3)$ and $n \in\{2,4\}$ for $\operatorname{BS}(2,7)$. The suffix of any geodesic in $\mathcal{M}$ is a word in the letters $b, B, T$. Each step of the McCann reduction algorithm takes place in the main upper half-sheet; thus, every $\gamma \in \mathcal{M}$ sits inside the main upper halfsheet and has the visual appearance of going straight up, across the cap, and then going down interspersed with horizontal shifts. (Benson Farb coined the term mesa for such a path, as it is the profile of a typical Utah redrock landform.) It is clear that any horizontal shifts in the suffix of $\gamma$ must occur as (multiples of) $b^{ \pm p}$ in order to avoid branching off the main sheet. Also, when $p \mid q$, a partial suffix for $\gamma$ can continue to go straight down without horizontal shifts and be guaranteed to stay on the main sheet (necessary and sufficient criteria for the suffix of $\gamma$ when $p \nmid q$ will be discussed in Section 6). Although it is not true that every possible geodesic for $b^{k}$ belongs to the set $\mathcal{M}$, it is true that for $k$ sufficiently large, every geodesic representing $b^{k}$ must belong to an upper half-sheet and travel up with limited horizontal shifts, have a horizontal cap, and then go down with limited horizontal shifts. In the upper half-sheet housing such a geodesic we define a stratum as the maximal (non-empty) segment of a horocycle in this sheet bounded by vertices of the geodesic. In particular, the cap of a geodesic is its highest stratum (see Figure 4.2) and in general every stratum is a path labeled $b^{l}$ for some $l>0$. In fact, all 
strata associated to a geodesic representing $b^{k}$ (except possibly the lowest stratum) must be paths labeled by a word of the form $b^{p l}$.

Using the McCann algorithm, one can tally the number of geodesics of fixed length $r=\left|b^{k}\right|$ as $k$ is varied. In this way, the growth series for the horocyclic subgroup of $\mathrm{BS}(p, q)$ can be partially constructed. The time complexity to compute $\left|b^{k}\right|$ is polynomial in $k$; however, the exponential growth of elements in the subgroup with respect to geodesic radius makes the tally of geodesics an unfeasible computation. Furthermore, when $p$ does not divide $q$ we have been unable to find a useful upper bound on the size of those exponents $k$ in terms of the given geodesic length $r=\left|b^{k}\right|$. Thus, to compute the first 100 terms (say) of the growth series for $\langle b\rangle$ via the McCann algorithm, one runs the program several times with various large exponent upper bounds and hopes that the 100th term stabilizes on the correct value. This unsatisfactory situation is ameliorated in Section 6 below.

It is visually obvious that the mesa shape of any $\gamma \in \mathcal{M}$ representing $b^{k} \in\langle b\rangle, k>0$ can be horizontally reversed. Indeed, the word $\gamma^{-1}$ is such a reversal and represents $b^{-k}$. Tracing the path labeled by $\gamma^{-1}$ from left to right represents a mirror image $\bar{\gamma}$ of the original mesa $\gamma$, yet is still a geodesic path for $b^{k}$. Equivalently, as a word $\bar{\gamma}$ is $\gamma^{-1}$ except that the letters $b, B$ are interchanged throughout the word. In this way, we obtain a second geodesic combing $\overline{\mathcal{M}}$ for the horocyclic subgroup. The prefix of $\bar{\gamma} \in \overline{\mathcal{M}}$ is a geodesic stem for the horocycle containing the cap of $\bar{\gamma}$. A major difference between $\mathcal{M}$ and $\overline{\mathcal{M}}$ is that geodesics from the latter set do not generally lie in the main upper half-sheet, but respectively reside in various different upper half-sheets. Observe that the combing $\mathcal{M}$ satisfies a fellow-traveller property, the reason being that the main upper half-sheet is quasi-isometric to a half-space in the hyperbolic plane. On the other hand, geodesics from the combing $\overline{\mathcal{M}}$ with close end points can lie in distinct sheets and radically diverge before getting close again.

The most basic method found for building sheets and computing distances in sheets has been termed the 'brick-laying' algorithm by Elder, who uses it to create upper half-sheets [10] (although we used the method as early as 2003, sans the very apt name coined by Elder). The idea is related to the McCann algorithm. The latter starts with a base stratum of $b^{L}$ and fills in horobricks to create ascending strata, whereas brick laying starts with a base of several horobricks, places copies of these horobricks above the originals, and fills in the gaps underneath the upper horobricks. We use brick laying in Lemmas 5.2 and 5.3 and more extensively in the following [14].

\section{The horocyclic subgroup: $p=2, q=4$}

For the balance of this section, we assume that $p>1$ and $p \mid q$. In Section 9 below, we show that the horocyclic subgroup has an unambiguous context-free geodesic combing, and in fact rational growth series. A more direct method is possible to create context-free grammars to generate the geodesics of the horocyclic subgroup. There are several distinct cases depending on the form of the quotient $\frac{q}{p}$. We illustrate some specifics in the case $p=2, q=4$ and summarize the results of the other cases in the appendix. (The derivations there were obtained using the much less transparent methods of Section 6 below.) Consider the combing $\mathcal{M}$ by McCann geodesics. Observe that all these geodesics can move sideways only by paths $b^{2}$ or $B^{2}$. As a consequence, any McCann geodesic that ends with $T$ will have even length. An even-length geodesic will always represent some $b^{2 r}$, while on the other hand if $b^{2 r}$ is represented by $\gamma \in \mathcal{M}$ then $\gamma$ has even length and ends in $T, b^{2}$ or $B^{2}$. The start of each suffix (the subword after the cap) for any length $\gamma \in \mathcal{M}$ is necessarily $T$. According to Figure 4.1, the maximum cap length for $\operatorname{BS}(2,4)$ ought to be four. However the conjugate $t b^{2} T$ is equivalent to $b^{4}$ and both have the same length, so we use $b^{2}$ as the sole geodesic cap for the case $p=2, q=4$.

Definition 5.1. Let $s$ be a suffix of a McCann geodesic $\gamma=t^{n} c s$ having even length of eight or more (where $c$ represents the cap). The (McCann) cone having stem at $\gamma$ is the set of all 


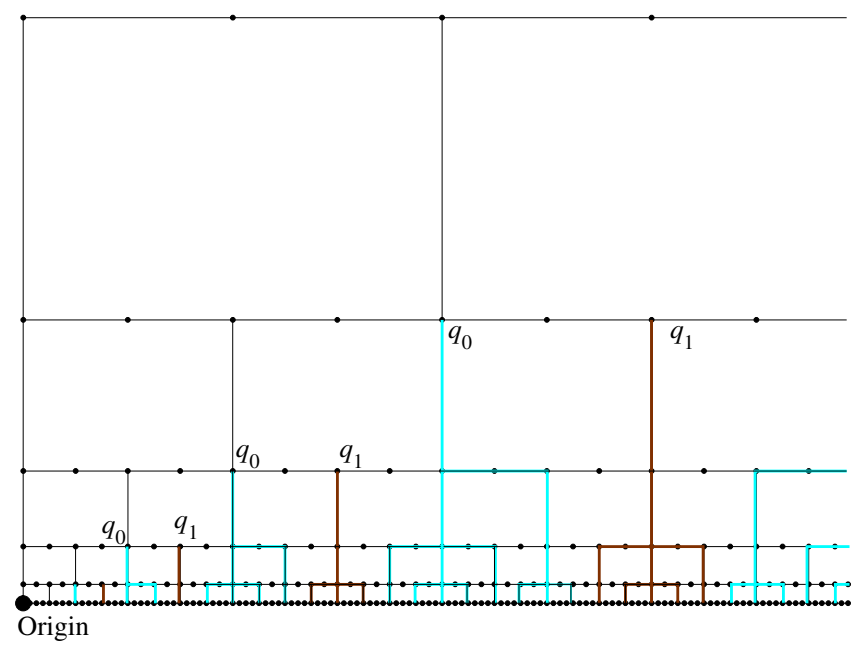

Figure 5.1. The two McCann cone types for BS(2,4), colored cyan and brown.

geodesic strings $u$ such that $t^{j} \gamma u=t^{j+n} \operatorname{csu}$ is a valid McCann geodesic, where $j$ is necessarily the number of occurrences of $T$ in the word $u$. Two cones $C, C^{\prime}$ with respective stems $\gamma, \gamma^{\prime}$ are defined to have the same cone type if $C=C^{\prime}$ as sets.

Examination of 'Quadrant I' of the main half-sheet for $\mathrm{BS}(2,4)$ reveals two McCann cone types (refer to Figure 5.1), which we designate $q_{0}$ and $q_{1}$. Type $q_{1}$ starts with two downward edges and then splits into three branches via a simple recursive pattern. The left-hand branch moves two edges left and then initiates a new $q_{1}$ cone. The center branch goes down one edge and spawns left and right paths that move sideways two edges and spawn new $q_{1}$ cones. The right-hand branch is a mirror image of the left-hand branch. The cone type $q_{0}$ looks just like type $q_{1}$ except for the addition of another $q_{1}$ type subcone after the edge path $T b b$. For technical reasons, we use a slightly more complicated decomposition. Type $q_{0}$ can be assembled from two subcones of type $q_{1}$ after edge paths of $T b b$ and $T T B B$, plus another type $q_{0}$ after edge path $T$. Type $q_{1}$ is built from a single $q_{0}$ after edge path $T$, plus a $q_{1}$ after edge path TTBB.

We develop a deterministic finite automaton (DFA) that accepts geodesic paths based on the above recursions. The start state implicitly represents a prefix of the form $t^{n} b b$, where $n>0$. The cone types $q_{0}$ and $q_{1}$ become accept states. From the start state, we can reach $q_{0}$ on letter $T$, and $q_{1}$ on word $T b b$. The recursion construction from the previous paragraph is incorporated to define transitions between $q_{0}$ and $q_{1}$. The next lemma shows that with just these three states the DFA accepts the suffixes $s$ for all McCann geodesics of the form $t^{n} b^{2} s$ $(n>1)$ having even lengths. As we remarked above, such geodesics will represent the even powers of $b$ from four on.

LEMma 5.2. Each vertex $b^{4 r}$ (respectively $b^{4 r+2}$ ) corresponds to the start of a McCann cone having type $q_{0}$ (respectively $q_{1}$ ) for all $r \geqslant 1$.

Proof. We use induction on $r$. When $r=1$, the vertices $b^{4}, b^{6}$ are the start points of cones with types $q_{0}, q_{1}$ respectively by fiat. Assume that the finite sequence of vertices $b^{4}, b^{6}, b^{8}, \ldots, b^{4(k-1)}, b^{4 k-2}$ are the start points of the corresponding alternating cone type sequence $q_{0}, q_{1}, q_{0}, \ldots, q_{0}, q_{1}$ for some $k>2$. Here the brick-laying idea outlined in [10] comes into play: the cosets $t^{n}\langle b\rangle, n>0$, are isometric copies of $\langle b\rangle$ and, in particular, the vertex $t b^{2 k}$ copies $b^{2 k}$ and is therefore the start of a McCann cone of either type $q_{0}$ or $q_{1}$ from our 

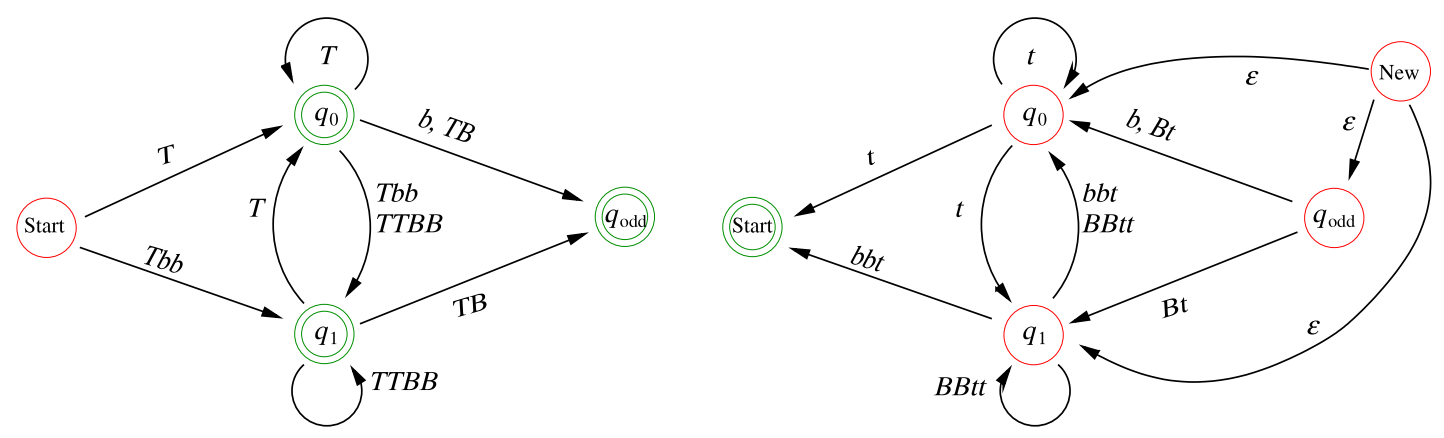

FiguRE 5.2. DFA based on cone types (left) and its reversal (right).

induction hypothesis (refer to Figure 5.1 throughout). Observe that $t b^{2 k}$ lies directly above $b^{4 k}$. The previously defined transitions $q_{0}, q_{1} \stackrel{T}{\longrightarrow} q_{0}$ show that $b^{4 k}$ corresponds to cone type $q_{0}$. If $t b^{2 k}$ has cone type $q_{0}$, then the existing transition $q_{0} \stackrel{T b b}{\longrightarrow} q_{1}$ shows that $b^{4 k+2}$ corresponds to cone type $q_{1}$. Lastly, if $t b^{2 k}$ has cone type $q_{1}$, we observe that $k$ is an odd integer by the induction hypothesis. Therefore, $2 k+2$ can be written as $4 j$ for some $j \geqslant 2$ and the vertex $t^{2} b^{2 j}$ has type $q_{0}$ or $q_{1}$ by the induction hypothesis. The existing transitions $q_{0}, q_{1} \stackrel{T T b b}{\longrightarrow} q_{1}$ show that $b^{4 k+2}$ corresponds to cone type $q_{1}$.

To accept suffixes for McCann geodesics with odd lengths (or, equivalently, geodesics which represent odd, positive powers of $b$ ), we add a new accept state $q_{\text {odd }}$ with transitions as shown in Figure 5.2 (left).

Lemma 5.3. The DFA of Figure 5.2 (left) accepts/produces suffixes $s$ such that the family $\left\{t^{n} b^{2} s\right\}$ (where $n$ is the number of T's in $s$ ) represents each $b^{k}, k \geqslant 4$.

Proof. Our previous argument shows that such suffixes are accepted when $t^{n} b^{2} s$ represents $b^{k}$ for any even integer $k \geqslant 4$. Let us consider the remaining cases when $k>4$ is odd.

When $k \equiv 1(\bmod 8)$ or $k \equiv 5(\bmod 8), b^{k}$ is then one unit right of the start of a type $q_{0}$ McCann cone corresponding to suffix $s^{\prime}$ that is accepted by the DFA. Observe that the existing transition $q_{0} \stackrel{b}{\longrightarrow} q_{\text {odd }}$ shows that $s=s^{\prime} b$ produces the desired suffix for $t^{n} b^{2} s$ which represents $b^{k}$.

When $k \equiv 3(\bmod 8), b^{k}$ then lies one unit left of the vertex $b^{2 i}$, where $2 i \equiv 4(\bmod 8)$ and $b^{2 i}$ is the start of a type $q_{0}$ McCann cone. Immediately above lies $t b^{i}$, where $i \equiv 2(\bmod 4)$; hence, $t b^{i}$ is the start of a $q_{1}$ type cone according to Lemma 5.2. Observe that the existing transition $q_{1} \stackrel{T B}{\longrightarrow} q_{\text {odd }}$ will produce the desired suffix for $t^{n} b^{2} s$ which represents $b^{k}$.

When $k \equiv 7(\bmod 8)$, the argument of the previous paragraph shows that $b^{k}$ lies one unit below and one unit left of a type $q_{0}$ vertex and the existing transition $q_{0} \stackrel{T B}{\longrightarrow} q_{\text {odd }}$ will produce the desired suffix for $t^{n} b^{2} s$ which represents $b^{k}$.

It is a tedious but elementary exercise that no two elements of the geodesic family $\left\{t^{n} b^{2} s\right\}$ are equivalent in $\mathrm{BS}(2,4)$. We leave this exercise to the reader. Our goal is to generate a grammar for the McCann geodesics, but directly using the machine of Figure 5.2 (left) will not work for this. The idea is to assign a prefix $t^{n} b b$ to the start state (with $n \geqslant 1$ to be determined later) and use the states as non-terminal symbols and the transitions as terminal symbols. The problem is that a grammar like this parses a word by simultaneously adding symbols to both ends. We want a grammar in which each parse step adds a contiguous block of symbols 


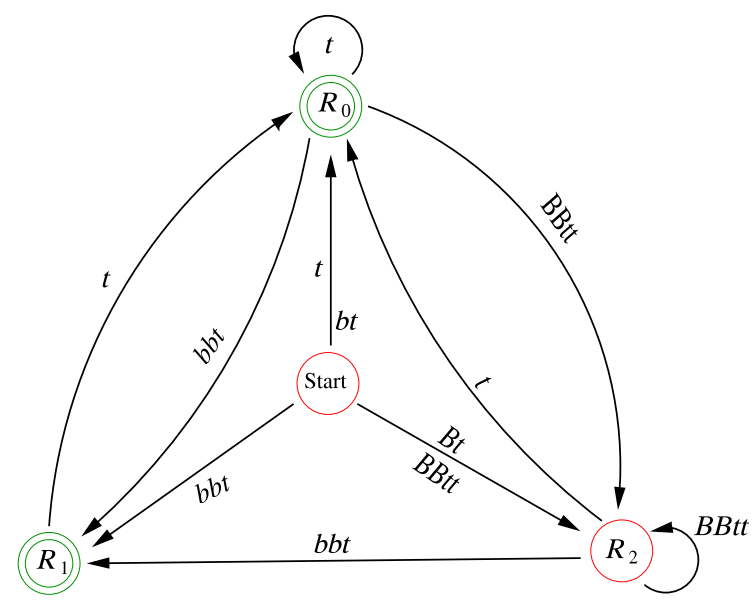

Figure 5.3. The minimal reversed DFA.

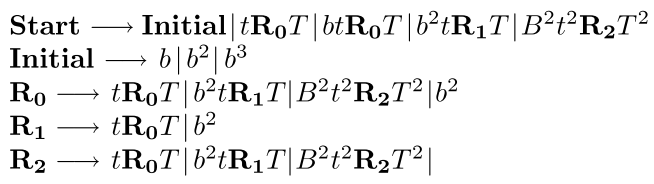

FiguRE 5.4. The resulting grammar.

in the middle of the word that is being built. To remedy this situation, we reverse the DFA of Figure 5.2 (left). In essence, this constructs the geodesic suffix by tracing backwards in the main half-sheet.

The standard recipe for obtaining the reversal of a DFA (see [17]) keeps the same states but reverses the transition arrows and words (and we will replace each $T$ with $t$ ). There is only one accept state, namely, the original start state. A new start state is created with new $\epsilon-$ transitions pointing to the original accept states. The machine is necessarily non-deterministic and is illustrated in Figure 5.2 (right).

It is evident that state $q_{\text {odd }}$ can be eliminated from the reversed DFA. In fact, we can reduce to the essentially unique minimal DFA that accepts the same reversal language [17]. The details are tedious and we employed the JFLAP software package (www.jflap.org) to help automate the process. The minimal DFA consists of four states, which we have named $R_{0}, R_{1}, R_{2}$, and (a new) Start. State $R_{0}$ is really the old cone type $q_{0}$. States $R_{1}$ and $R_{2}$ form a splitting of the old cone type $q_{1}$. The machine is shown in Figure 5.3 and can produce a grammar for a reverse geodesic combing, at least for the words $b^{r}, r \geqslant 4$. The transitions from the Start state represent a geodesic path starting at some $b^{r}$ (there are some initial exceptions for $r \in\{1,2,3\}$ ) that travels up through various cones until the transitions end in an accept state. The latter represents the cap $b b$ followed by a strictly vertical path back to the origin. Now it becomes clear why we need a new non-accept state $R_{2}$ that is a copy of $R_{1}$ : we cannot accept the non-geodesic $B^{2} t^{2} b^{2} T^{2}$ when we already have the equivalent $b^{2} t b^{2} T$. The grammar is displayed in Figure 5.4.

It should be evident that this grammar is unambiguous, since the underlying DFA is unambiguous. A theorem of Chomsky-Schützenberger (see [13] for example) implies that the growth series for this language is algebraic, and provides a method to explicitly compute the growth. We replace each production arrow with equality, all terminals become the variable $z$, '।' becomes ' + ', and juxtaposition becomes (commutative) multiplication. This yields a system of equations where the non-terminal symbols are variables as in Figure 5.5. The system is solved 


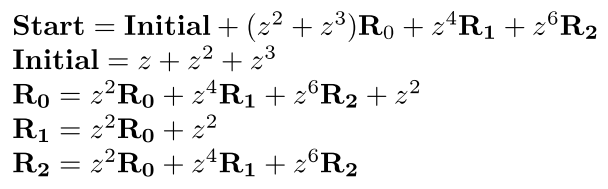

FIGURE 5.5. Chomsky-Schützenberger grammar equations.

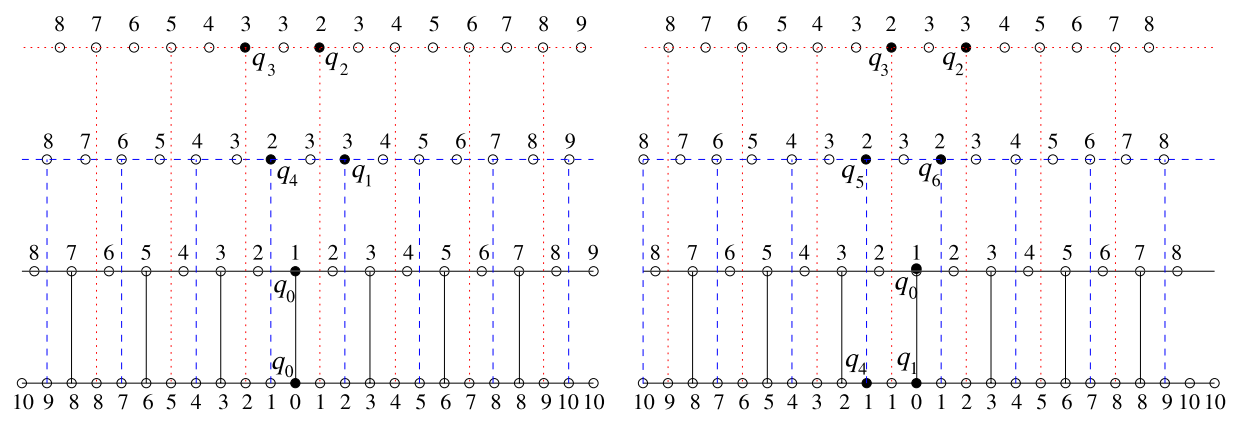

FiguRE 6.1. Branching of upward horocycle types; 6.1(a) (left) and 6.1(b) (right). Distances are relative to the lowest horocycle of each sketch.

for Start, which equals a closed form for the growth series as a function of $z$. We obtain

$$
\operatorname{Start}(z)=\frac{z\left(1+z+z^{5}-z^{6}-z^{7}\right)}{1-z^{2}-2 z^{6}}
$$

as the growth function for $\left\{b^{r}: r \geqslant 1\right\}$. The growth for the entire horocyclic subgroup is $B(z)=1+2 \operatorname{Start}(z)$.

\section{The horocyclic subgroup: $p=2, q=3$}

We first focus on the simplest concrete example where $p \nmid q$, namely $\mathrm{BS}(2,3)$. To construct a geodesic combing of the horocyclic subgroup, we start with a finite-state automaton for building geodesic stems for upper horocycles.

An up-point $u$ is a group element represented by a geodesic word in the generators $b, B, t$ and ending in $t$ such that no other geodesic that ends in $T, b$, or $B$ can represent $u$. Define a horocycle $K$ to be upper if some geodesic stem for $K$ contains no instances of the letter $T$. A closest point projection or relative origin (cf. Section 3 above) for $K$ is a vertex $v$ such that the metric distance between the origin and $K$ is realized by $|v|$.

Every relative origin of an upper horocycle is an up-point, but not conversely. Both $B t$ and $b b t$ are up-points for the coset $t\langle b\rangle$, but only the former is a relative origin. There are five equivalence classes (see Section 8 for a formal definition) of upper horocycles, counting leftright mirror images as distinct classes in $\mathrm{BS}(2,3)$. These classes and their upward transitions are derived in Figures 6.1(a) through 6.2(b). Observe that every relative origin for an upper horocycle $K$ has a geodesic stem that passes through an up-point of the nearest upper horocycle beneath $K$. In fact, the up-points can be classified into exactly eight distinct types (these are in fact upward cone types as per Corollary 8.3) depending upon what upper horocycles lie above them. We use the eight types and the transition edges connecting them to construct an eight-state automaton that accepts various paths. We will then eliminate several possible transitions (for example, we omit the transition $b t$ from $q_{4}$ to $q_{3}$ ) so as to produce unique paths.

Proposition 6.1. The finite-state automaton of Figure 6.3 constructs geodesic paths only, and every possible up-point is the terminus of some accepted path. 

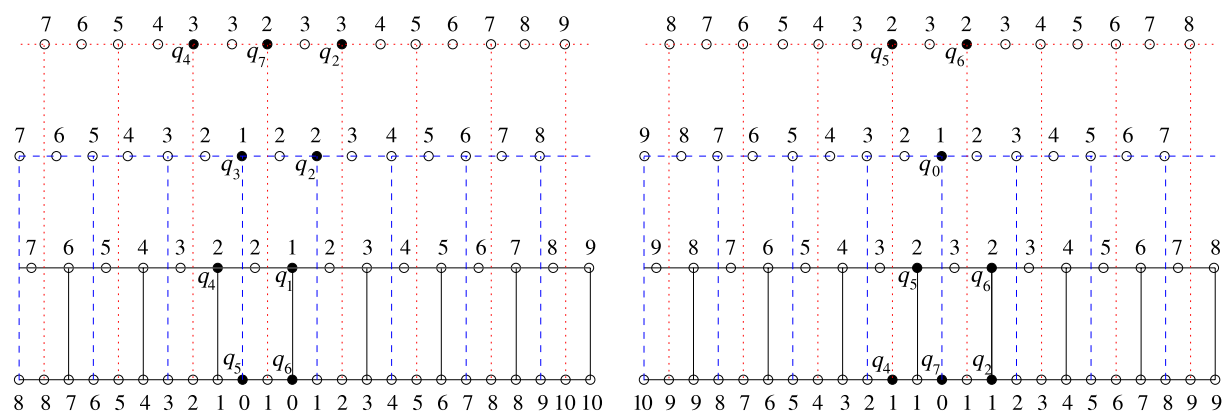

Figure 6.2. The remaining upward horocycle types; 6.2(a) (left) and 6.2(b) (right).

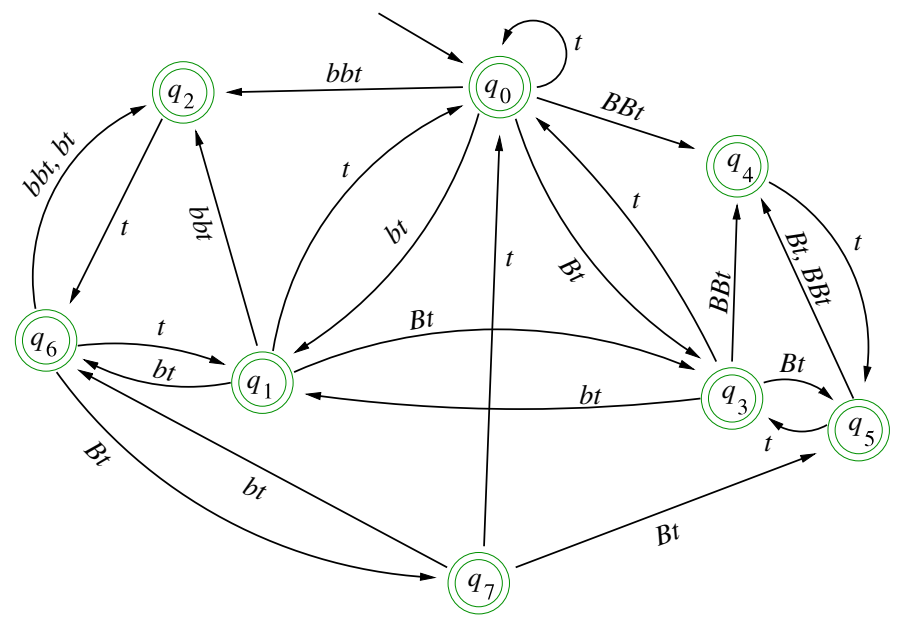

Figure 6.3. The initial DFA based on up-points.

Proof. We do an induction on the number of transitions in the machine (equivalently, on the $t$ exponent sum of the output word). The paths $t, b t, B t, b b t, B B t$ are all geodesics and end in all the possible up-points immediately above the horocyclic subgroup. Assume, for some $k \geqslant 2$, that every accepted word produced by $k-1$ transitions is geodesic and all uppoints at this $t$ level are end points of this set of paths. Let $w$ be accepted after exactly $k$ transitions. By construction, $w$ will have the form $w=u s$, where $s \in\{t, b t, B t, b b t, B B t\}$ and $u$ is a geodesic terminating in some up-point. If $w$ is not geodesic, then there is a shorter word from the origin with the same terminus as $w$. Both these paths project to the same arc in the Bass-Serre tree (side view) of $\mathrm{BS}(2,3)$, which means that both paths go through the same penultimate horocycle beneath $w\langle b\rangle$. The supposed shorter word must have the form $u^{\prime} s^{\prime}$, where $s^{\prime} \in\{t, b t, B t, b b t, B B t\}$ and $u^{\prime}$ necessarily ends in an up-point with $t$ exponent sum of $k-1$. By the induction hypothesis, $u^{\prime}$ has been constructed by our machine after $k-1$ transitions. This implies that the end points of $\left(u, u^{\prime}\right)$ coincide with the state pairs $\left(q_{4}, q_{1}\right)$ or $\left(q_{2}, q_{3}\right)$ or a pair from $\left\{q_{2}, q_{4}, q_{7}\right\}$. Examination of Figures 6.1(a) through 6.2(b) shows that the length of $u^{\prime}$ is at most one edge less than the length of $u$ and that there is no combination of suffixes $s, s^{\prime}$ that enables both $u s$ and $u^{\prime} s^{\prime}$ to end at the same up-point with $u^{\prime} s^{\prime}$ shorter than $u s$.

We will only be concerned with geodesic paths for $b^{r}, r>0$, as horizontal symmetry will reproduce the others. Observe in Figures 6.1(a) through 6.2(b) that the up-points $q_{3}, q_{4}, q_{5}, q_{7}$ are always to the left of one of $q_{1}, q_{2}, q_{6}$ on any given upper horocycle. Adjoining a rightward 


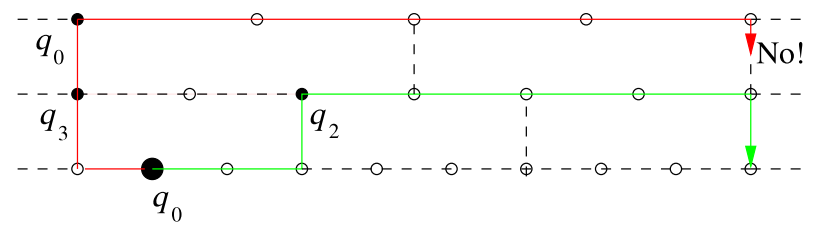

Figure 6.4. Adding caps creates complications: the red path should be rejected in favor of the green path.

cap of $b^{4}$ to any up-point in the former group will always produce a longer path than adjoining the same cap to one of the latter up-points. Thus, we change $q_{3}, q_{4}, q_{5}, q_{7}$ into non-accept states; the machine still outputs geodesics. To each geodesic produced by the (modified) finite-state automaton we add a cap of $b^{4}$ and suffixes of the form $T^{*}$. The actual number of $T$ 's will balance the number of $t$ 's in the stem. There are a couple of alterations needed to the finitestate machine once we consider adding the cap $b^{4}$ after reaching an accept state. For instance, in testing the string BttbbbbTT that represents $b^{8}$, the machine starts in state $q_{0}$ and transitions to state $q_{3}$ and back to $q_{0}$. The latter is ostensibly an accept state so the cap is added; however, $b^{8}$ is also represented by the accepted shorter path $b^{2} t b^{4} t$ (see Figure 6.4).

Similarly, $b^{20}$ is represented by two words bbttbbtbbbbTTT and bbttBttbbbbTTTT with accepted up-point prefixes but only the former is geodesic. The latter has the following transition sequence: $q_{0} \rightarrow q_{2} \rightarrow q_{6} \rightarrow q_{7} \rightarrow q_{0}$ then $b^{4}$ cap.

We observe in general that the addition of the cap can yield non-geodesic or duplicate geodesic paths that will be ostensibly accepted, but only according to a certain pattern: consider a pair of geodesics $\gamma_{1}, \gamma_{2}$ from the origin to $b^{r}, r>6$, that might be constructed from our DFA (see Figure 8.2 for a schematic of this scenario). Without loss of generality, we may assume that $\gamma_{1}$ is generally to the left and/or above $\gamma_{2}$ and both lie in a common upper half-sheet. Each of these geodesics will start as a concatenation of up-point paths as illustrated in Figures 6.1 and 6.2. Note that such up-point paths can take opposite sides of at most one horobrick at each $t$-level (this also verifies that $\gamma_{1}$ and $\gamma_{2}$ reside in a common upper half-sheet). The elimination of duplicate paths prior to Proposition 6.1 above shows that our DFA prevents $\gamma_{1}, \gamma_{2}$ from reconnecting during the up-point/prefix phase of construction. Thus, Figure 8.1 is true in the sense that the cap for $\gamma_{1}$ must be at a higher level than the cap for $\gamma_{2}$, in fact exactly one level higher in the current situation.

We see that the highest up-point on $\gamma_{2}$ and the next-to-highest up-point for $\gamma_{1}$ sit on the same horocycle and must, by Figures 6.1 and 6.2, comprise one of the pairs $\left(q_{3}, q_{2}\right),\left(q_{4}, q_{1}\right),\left(q_{5}, q_{6}\right)$, or a pair from $\left\{q_{4}, q_{7}, q_{2}\right\}$. We begin with any pair where $q_{4}$ comprises the leftmost up-point. There are no up-point continuations from $q_{4}$ to any other accept state (see Figures 6.1(b) and 6.2(b)); such a pair presents no problems. By the same reasoning, no pair having $q_{5}$ as leftmost up-point poses a problem (Figure 6.2(a)). The pairs $\left(q_{3}, q_{2}\right)$ and $\left(q_{7}, q_{2}\right)$ can and do present unresolved problems as shown above with the concrete examples concerning $b^{8}$ and $b^{20}$.

The fix to the DFA is now described. Create a new state $q_{8}$ that is a copy of $q_{0}$ and replace the $t$ labeled transitions $q_{3} \rightarrow q_{0}$ and $q_{7} \rightarrow q_{0}$ with $t$ transitions $q_{3} \rightarrow q_{8}$ and $q_{7} \rightarrow q_{8}$. Do not copy to $q_{8}$ the $t$ loop $q_{0} \rightarrow q_{0}$ and, finally, change $q_{8}$ to fail status. The modified machine is illustrated in Figure 6.5.

We now have a formula to generate geodesics for elements of the horocyclic subgroup in BS $(2,3)$ : use the DFA of Figure 6.5 to generate an accepted prefix $v$ of up-point paths and add a cap $b^{4}$ and a suffix $T^{k}$, where $k$ is the number of $t$ 's in the prefix $v$. Most of the paths created in this construction do not represent words of the form $b^{r}$. Geometrically (in the Bass-Serre tree side view of the Cayley graph), the suffix $T^{k}$ diagonals away from the horocyclic subgroup. A necessary criterion for a path of the indicated form to end on the horocyclic subgroup is 


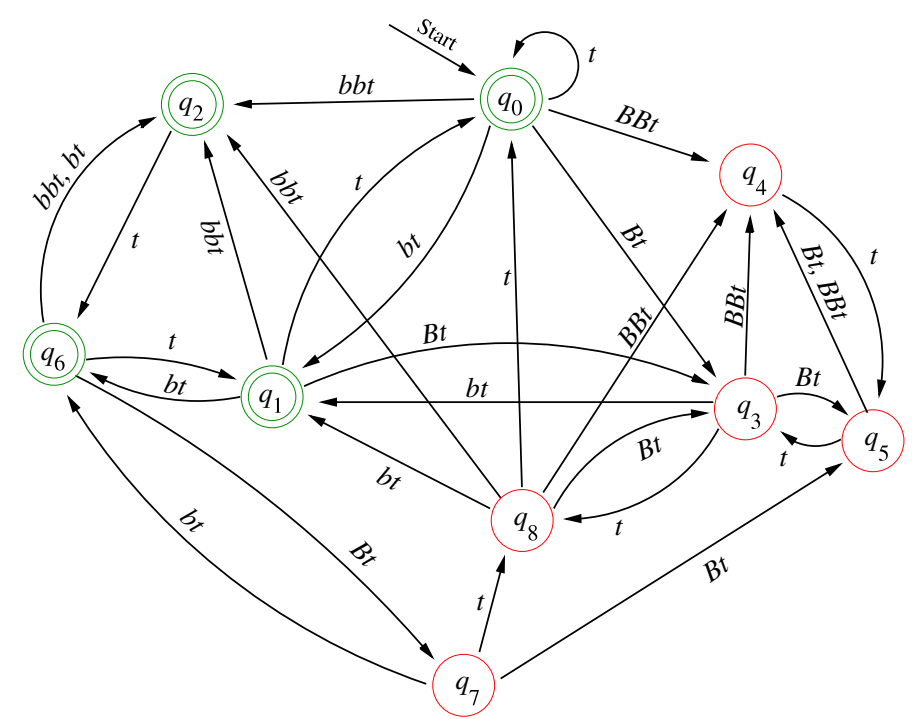

Figure 6.5. The modified DFA.

that each stratum (except possibly the lowest) associated to the path has the form $b^{l}$ with $l$ an even integer. We test the parity of each strata length (except the lowest stratum) for each of the constructed $v b^{4} T^{k}$ and keep only those with even length.

We denote the paths generated by this procedure as the set $\mathcal{S}$ of Schofield geodesics. There are similarities between the Schofield combing $\mathcal{S}$ and the reverse McCann combing $\overline{\mathcal{M}}$, but in general they are not the same set of paths. For a given $r>6$, both the Schofield and the reverse McCann geodesics representing $b^{r}$ trace a 'backwards' path and lie in a common upper half-sheet $H$. However, their distinct methods of construction allow the respective paths to take different routes around relators in $H$. The so-called Schofield algorithm automates the construction and testing of the geodesics in $\mathcal{S}$, numerically keeping track of path lengths. This is a more satisfactory method to compute growth coefficients than the McCann algorithm because the process loops over geodesic radii. (Serial and parallel versions of the Schofield algorithm are available for download at [18].)

Proposition 6.2. There is a unique Schofield geodesic representing $b^{r} \in \mathrm{BS}(2,3)$ for each $r>6$.

Proof. Uniqueness follows from the unique prefixes generated by the finite-state machine, and the fact that the cap assignments were modified to eliminate duplication. Recall that the set $\overline{\mathcal{M}}$ of mirrored McCann geodesics forms a combing for $\langle b\rangle$, so that there is some $\bar{w} \in \overline{\mathcal{M}}$ that represents $b^{r}$, where $\bar{w}=v b^{4} T^{k}$ has $v$ as an upward geodesic stem for the coset $v\langle b\rangle$. The prefix $v$ must obviously terminate in an up-point. Proposition 6.1 shows that this up-point is also the end point of a geodesic $u$ accepted by the finite-state machine of Figure 6.5. This up-point cannot correspond to any of the states $q_{3}, q_{4}, q_{5}, q_{7}$ since $v$ could then be replaced by an up-point to the right, which would shorten $\bar{w}$. We conclude that the up-point is of type $q_{0}, q_{1}, q_{2}$, or $q_{6}$, whence $u$ is produced by the modified automaton of Figure 6.5. We deduce that $v$ and $u$ represent the same group element, have the same length, and therefore $u b^{n} T^{k} \in \mathcal{S}$ is a geodesic representative for $b^{r}$ (in fact, $v$ and $u$ can be, but are not generally, the same word).

We describe a deterministic single-tape Turing machine that accepts the Schofield language for $\mathrm{BS}(2,3)$. A realization of this machine, written by Jared Adams and Chris McGahan 
using JFLAP, is available for download at [18]. The tape alphabet consists of the symbols $b, B, t, T, 0,1$ along with begin-word, end-word, and padding symbols ${ }^{\wedge}, \$$, and $\mid$ respectively. We incorporate the finitely many states needed to check that a given word belongs to the Schofield family of geodesic stems. (This is done by rewriting the DFA of Figure 6.5 so that transitions are restricted to the reading of single symbols instead of the words shown in Figure 6.5.) An input word is read where it is verified (if any verification step fails, the machine fails to accept) that the word comes from ${ }^{\wedge}\{b, B, t, T\}^{*} \$$, and has the form stem-cap-suffix, where the stem comes from the DFA of Figure 6.5 and cap + suffix has the form $b b b b T^{*} \$$.

Upon reading the end-tape symbol $\$$ the tape is read backwards until the cap $b b b b$ is reached. All steps to this point are accomplished via finitely many control states and no writing to the tape. Starting from the right of this cap, the first symbol $T$ is over-written by $\mid$. The tape is read right until $\$$ is encountered and replaced with $T$ and $\$$ written to its right. The machine reads left and replaces the cap $b b b b$ with $\mid 100$ (this is the binary version of 'four' with a leading padding symbol). It checks left to ensure that there is a $t$ and goes back right and erases the rightmost $T$ and moves $\$$ one cell left. The binary block is multiplied by 1.1 (this is the binary version of $\frac{3}{2}$ ) and the rightmost $t$ becomes $\mid$. The machine proceeds to alternately keep track of $t T$ pairs and replacing these letters with padding symbols, and do arithmetic to the binary block based on the letters of the geodesic stem. If the symbol is $b$ (respectively $B$ ), then replace with $\mid$ and add 1 to (respectively subtract 1 from) the binary block. When the stem symbol read is $t$, multiply the binary block by 1.1. As necessary, the padding symbols can be replaced with additional binary placeholders (observe that at most one new placeholder is needed for each letter read). Repeat reading symbols to the left of the binary block and doing binary arithmetic as per the conditions above until multiplication by 1.1 fails to produce an integer (reject) or $\$$ is read (accept). Observe that the binary mathematics computes successive strata lengths and the final number $r$ displayed for an accepted string $w$ is the exponent (lowest stratum) for which $w$ is equivalent to $b^{r}$.

Corollary 6.3. The Schofield language of geodesics is context sensitive and is accepted with quadratic time complexity.

Proof. The Turing machine described above (and implemented at [18]) needs only $n+3$ tape cells (this includes the special symbols ${ }^{2}, \$, \mid$ ) on an input string of length $n$ in order to accept/reject. By [17], this language is context sensitive. The number of tape-head moves can also be estimated. There is an initial forward read, then backwards to the cap which uses less than $2(n+3)$ moves. The binary arithmetic uses left and right passes, each of which needs less than $n+3$ moves. The number of these passes is bounded by the length of the input string, so in the worst-case scenario the total number of head moves is less than $2(n+3)+n(n+3)$.

\section{When $p$ does not divide $q$ : generalizations}

We begin with the following number theoretic result due to Andreas Weingartner. Let $\alpha(x)=\frac{q}{p} x$, where $p$ and $q$ are relatively prime integers and $q \geqslant 2$. Define $\beta_{+}(x)=x+1$ and $\beta_{-}(x)=x-1$. Let $\varphi(x)$ be any finite composition of the three functions $\alpha, \beta_{+}$, and $\beta_{-}$that includes at least one instance of $\alpha$. We write $\varphi^{n}$ to mean the $n$-fold iteration of the function $\varphi$.

Lemma 7.1 (Weingartner). Suppose that $x_{0}$ is any positive integer. The sequence $x_{n}=$ $\varphi^{n}\left(x_{0}\right)$ is either constant or it will contain non-integer values.

Proof. We may write $\varphi$ as

$$
\varphi(x)=\frac{q}{p}\left(\ldots\left(\frac{q}{p}\left(\frac{q}{p}\left(\frac{q}{p}\left(x+d_{0}\right)+d_{1}\right)+d_{2}\right)+d_{3}\right) \ldots\right)+d_{k},
$$


where $k$ is the number of occurrences of $\alpha$ in $\varphi$, and each $d_{i}$ is an integer for $0 \leqslant i \leqslant k$. We can write (7.1) more simply as $\varphi(x)=a x+d$, where $a=(q / p)^{k}$ and $d$ is some rational number. Since $x_{n+1}=\varphi\left(x_{n}\right)=a x_{n}+d$, we have

$$
x_{n+1}-x_{n}=\left(a x_{n}+d\right)-\left(a x_{n-1}+d\right)=a\left(x_{n}-x_{n-1}\right) .
$$

By iterating (7.2), we obtain

$$
x_{n+1}-x_{n}=a^{n}\left(x_{1}-x_{0}\right)=\frac{q^{n k}\left(x_{1}-x_{0}\right)}{p^{n k}} .
$$

If $x_{1}=x_{0}$, then $\left\{x_{n}\right\}$ is a constant sequence. Assume $x_{1} \neq x_{0}$. If $x_{1}$ is not an integer, there is nothing to prove. If $x_{1}$ is an integer, let $p^{l}$ be the largest power of $p$ that divides $x_{n+1}-x_{n}$. Since $p$ and $q$ are relatively prime, (7.3) shows that $x_{n+1}-x_{n}$ is not an integer for all $n$ such that $n k>l$. For the smallest such $n$, we conclude that $x_{n+1}$ cannot be an integer since $x_{n}$ is.

We observe that the hypothesis of $p$ and $q$ being relatively prime can be weakened to $p \nmid q$, and the conclusion of Weingartner's lemma still holds. We think of $x_{0}$ as the path length along a cap $b^{x_{0}}$ on a mesa-type geodesic. The function $\alpha$ applied to $x_{0}$ measures the path length of the stratum belonging to $t b^{x_{0}} T$. The function $\beta_{+}$(respectively $\beta_{-}$) corresponds to altering path-length measurement after attaching a prefix $b$ (respectively $B$ ) to a stratum. Recall that each such stratum (except perhaps the lowest) from a valid mesa-type geodesic must have the form $b^{p k}$ for some $k>0$.

Theorem 7.2. Assume $p \nmid q$. Any combing of $\langle b\rangle$ by geodesics does not comprise a context-free language.

Proof. We use the pumping lemma for context-free languages [17]. Suppose $g_{1}=v w x y z$ is a sufficiently long geodesic word representing $b^{r}$ (with $r \gg 0$ ), where $v, w, x, y, z$ are subwords and not both of $w, y$ can be the empty string. If $g_{1}$ belonged to a context-free language, then $g_{n}=v w^{n} x y^{n} z$ would belong to the same language for all $n \geqslant 0$. One of the necessary conditions for $g_{n}$ to be a geodesic representative of a word in $\langle b\rangle$ is that $g_{n}$ have a generic mesa shape with the net exponent sum on the stable letter $t$ being zero. It follows that $w \in\{t, b, B\}^{*}$ and $y \in\{T, b, B\}^{*}$ with the number of $t$ 's in $w$ being the same as the number of $T$ 's in $y$ and this number is necessarily positive (else $w^{n}$ consists of arbitrarily long strings of $b$ 's or $B$ 's). For simplicity, we initially assume that $x$ represents a cap of the form $b^{s}$ for some $s \geqslant 0$.

In this case, we can write $g_{1}$ as a word of the form

$$
v \ldots\left(t\left(\ldots\left(t\left(b^{s}\right) T\right) \ldots\right) T\right) \ldots z,
$$

where the parentheses are nested according to the various strata of $g_{1}$ and can contain $b$ 's and/or $B$ 's. Here $\left(b^{s}\right)$ represents the cap of $g_{1}$, while $\left(t\left(b^{s}\right) T\right)$ is a representation of a word in the stratum immediately below the cap, etc. Since $g_{1}$ is assumed to be a geodesic for $b^{r}$, each of these strata is a word equivalent to a power of $b$ with exponent divisible by $p$ (with the possible exception of the lowest stratum $b^{r}$ ). Consider the growth of the exponent on $b$ as we descend the sequence of strata, starting with the cap. Each nested pair $t(\ldots) T$ acts as the function $\alpha$ on this exponent, as per Weingartner's lemma. Occurrences of $b$ (respectively $B$ ) act as $\beta_{+}$(respectively $\beta_{-}$). Thus, the subword $w x y$ of $g_{1}$ acts as the function $\varphi$ on the initial cap exponent of the mesa. As $n$ increases, this action is identical to that of the $n$-fold iteration $\varphi^{n}$. Weingartner's lemma shows that there will always be some $n$ for which the stratum $b$-exponent is not an integer. Prior to this $n$, there is a stratum whose path length is integral but fails to be a multiple of $p$. The geometric interpretation is that some $g_{n}$ branches away (downwards) from any upper half-sheet containing $g_{1}$ and cannot represent an element of the horocyclic subgroup. 
In the general case, it is possible that the middle subword $x$ contains $t$ 's and/or $T$ 's, with the exponent sum balanced by other $T$ or $t$ combinations in either or both of $v, z$. In this event, the cap of $g_{1}$ is no longer confined to $x$, but must include parts of $w$ and/or $y$. However, the latter two subwords must still have balanced exponent sum on the stable letter (else $g_{n}$ will fail to do so for various $n$ ). The construction of $\varphi^{n}$ and conclusion of the previous paragraph still obtain after offsetting the cap and strata across $w x y$.

Note that the previous proof is much stronger than is necessary for the conclusion of Theorem 7.2. Only a single violation of the pumping lemma is required, whereas we showed that every sufficiently long geodesic cannot be pumped indefinitely. It is not hard to imagine that geodesic combings of the horocyclic subgroup are far removed from the class of contextfree languages when $p \nmid q$. In fact, there are several formal language classes sandwiched between the context-free family and the context-sensitive family of the Chomsky hierarchy. One such class are termed the tree-adjoining languages generated by the tree-adjoining grammars (equivalently, the linearly indexed grammars or the head grammars [20]). Analogous to the context-free situation, there is a pumping lemma for this class [19].

Lemma. Every sufficiently long word in a tree-adjoining language can be written in the form $x w_{1} v_{1} w_{2} y w_{3} v_{2} w_{4} z$ with not all $w_{1}, w_{2}, w_{3}, w_{4}$ being the empty string and $x w_{1}^{n} v_{1} w_{2}^{n} y w_{3}^{n} v_{2} w_{4}^{n} z$ is in the same language for all $n>0$.

Corollary 7.3. Assume $p \nmid q$. Any combing of $\langle b\rangle$ by geodesics does not comprise a tree-adjoining language.

Proof. Let $g_{1}=x w_{1} v_{1} w_{2} y w_{3} v_{2} w_{4} z$ and $g_{n}=x w_{1}^{n} v_{1} w_{2}^{n} y w_{3}^{n} v_{2} w_{4}^{n} z$ for each $n \geqslant 0$. The proof of Theorem 7.2 implies that for all large enough $n>0$ the word segment $w_{2}^{n} y w_{3}^{n}$ contains the geodesic cap of $g_{1}$ and as a path departs from any half-sheet containing $g_{1}$. Thus, the suffix of $g_{n}$ (regardless of the choice of words $v_{2}, w_{4}, z$ ) will follow a different downward sheet than that of its prefix and miss the horocyclic subgroup entirely.

Recall the definition of cones on the main sheet from Section 5 . There we showed that the main upper half-sheet for $\operatorname{BS}(2,4)$ had only two basic (McCann) cone types. This is not true in general.

Corollary 7.4. Assume $p \nmid q$. The main half-sheet of $\operatorname{BS}(p, q)$ contains infinitely many McCann cone types.

Proof. Suppose that there are only finitely many such cone types. Then there will be at least one cone $\Gamma$ which contains a subcone $\Gamma^{\prime}$ having the same type. More explicitly, there will be a McCann geodesic word $t^{n} c s$, where $c$ is the cap and $s$ is the suffix that forms the stem for $\Gamma$, and the stem for $\Gamma^{\prime}$ will be a longer McCann geodesic having the form $t^{n+m} \operatorname{csu}$, where $m$ is the number of occurrences of $T$ in the word $u$. But then we can continue to 'pump' and obtain an infinite nesting of cones with valid McCann geodesics $t^{n+m i} \operatorname{csu} u^{i}, i>0$ as stems for these cones, which contradicts the proof of Theorem 7.2.

Observe that this proof is also stronger than the conclusion of Corollary 7.4: it shows that no cone of type $\mathcal{C}$ can contain any subcone of the same type $\mathcal{C}$, but can only contain completely different types as subcones.

\section{The horocyclic subgroup for arbitrary $p$ and $q$}

Suppose that $g \in \operatorname{BS}(p, q)$ is a vertex on some horocycle $K=w\langle b\rangle$, where $w$ is a geodesic stem. Recall from Section 6 the definition of up-point (which generalizes for all $1<p<q$ ) and from 


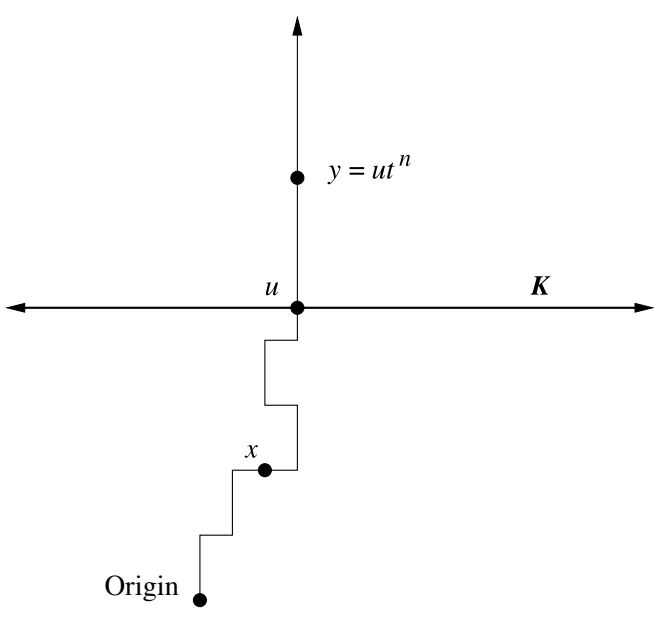

FiguRE 8.1. The vertical-tending ray is uniformly quasi-geodesic.

Section 3 that $|g|-|w|$ is the relative radius assigned to $g$ as used in the computation of the coset growth series for $K$ when the terminal vertex of the stem $w$ is a relative origin for $K$ (that is, has relative radius zero and coincides with a closest point projection to the origin). Imagine taking another horocycle $K^{\prime}$ and rigidly superimposing it on $K$ so that a relative origin for $K^{\prime}$ is glued to a relative origin for $K$. As per the level idea of Section 3, we define $K$ and $K^{\prime}$ to be equivalent if there is some such rigid superposition that matches relative radii for all vertices on the horocycles. This forms an obvious equivalence relation on horocycles, and equivalent horocycles will have the same relative growth function. As an example, $t\langle b\rangle$ and $\langle b\rangle$ are equivalent, and in fact for each $n \geqslant 0$ the cosets $t^{n}\langle b\rangle$ are all equivalent. We will show that for any fixed $\operatorname{BS}(p, q)$ there are only finitely many equivalence classes of upper horocycles (regardless of whether $p$ divides or fails to divide $q$ ).

Proposition 8.1. Suppose $K=w\langle b\rangle$ is an upper horocycle in $\operatorname{BS}(p, q)$. Then there is a constant $N>0$ depending only on $p$ and $q$ such that $K$ has at most $N$ up-points.

Proof. Let $K$ be an upper horocycle and $u \in K$ any up-point. Consider the ray starting at the origin $\mathcal{O}$, passing through $u$, and continuing straight up. The ray consists of a geodesic prefix (that defines $u$ ) and a geodesic suffix of the form $t^{\infty}$. Observe that this ray defines a unique upper half-sheet. It is entirely likely that the ray is in fact geodesic; however, for our purposes it is sufficient that it be uniformly quasi-geodesic.

To this end, let $x, y$ be any points on the ray. If both $x, y$ lie on the geodesic prefix, or both lie on the suffix, then $\overline{x y}$ is geodesic. Thus, we may suppose that $x$ lies in the prefix $\overline{\mathcal{O} u}$ and that $y=u t^{n}$ for some $n>0$ (see Figure 8.1). Let $m>0$ denote the number of vertical edges on the subpath $\overline{x u}$, and we have already defined $n$ as the number of vertical edges in $\overline{u y}$. The path $\overline{x u}$ may have horizontal segments between vertical edges; however, the longest such segment is less than $q$ units across (otherwise the segment $b^{ \pm q} t$ can be replaced by the shorter $t b^{ \pm p}$, respectively). We obtain the length estimate length $(\overline{x u})<q m$ and hence length $(\overline{x y})<q m+n<q(m+n)$.

On the other hand, the geodesic distance from $x$ to $y$ is at least $m+n$, by ignoring any horizontal edges. Therefore,

$$
\text { length }(\overline{x y}) \geqslant \operatorname{dist}(x, y) \geqslant m+n=\frac{1}{q} \cdot q(m+n)>\frac{1}{q} \cdot \operatorname{length}(\overline{x y}) .
$$

Since $x, y$ were arbitrary, this shows that the entire ray is quasi-geodesic $(q)$. 
Now use the standard quasi-isometry to embed the entire upper half-sheet defined by our ray into the hyperbolic plane (Poincaré upper half space model), so that the origin of the Cayley graph is pasted to the euclidean coordinates $(0,1)$. The multiplicative and additive constants of this quasi-isometry depend only on $p$ and $q$. The image of our ray is a quasi-geodesic hyperbolic ray emanating from $(0,1)$ and tending to the ideal boundary point at upper infinity. The hyperbolic geodesic ray $R=(0, y): y \geqslant 1$ shares the same initial point and tends towards the same ideal point. Standard hyperbolic geometry implies that there is some $\epsilon>0$ (depending on the new quasi-geodesic constant, which in turn depends only on $p$ and $q$ ) such that the quasigeodesic ray lies within the $\epsilon$-neighborhood of $R$. In particular, the embedded image of the up-point $u$ is in this neighborhood. Observe that the ray $R$ is perpendicular to the embedded image of horocycle $K$, and only that segment of the embedded $K$ within $\epsilon$ of $R$ can contain embedded up-points. In this situation, the hyperbolic arc length of this horocycle segment is proportional to $2 \epsilon$ (regardless of the height of the embedded $K$ ). Applying an inverse to the quasi-isometry gives a uniformly bounded segment of $K$ that contains all up-points, with the bound $N$ depending only on $p$ and $q$.

We remark that for lower horocycles (those with geodesic stem not containing $t$ ), the above argument fails. We observe (and leave to the reader to verify) that for every integer $m$ there is some lower horocycle with at least $m$ relative origins.

Proposition 8.2. For any fixed $1<p<q$, there are only finitely many equivalence classes of upper horocycles.

Proof. Consider the uniformly bounded zones on upper horocycles established in the previous proof. Such a zone has only finitely many possible configurations of up-points, and since relative radii of adjacent vertices can differ by at most one unit, there are only finitely many relative radii assignments possible for such a zone. Observe that if the upper horocycle $K^{\prime}$ lies immediately above the horocycle $K$, then any minimal geodesic stem $w^{\prime}$ for $K^{\prime}=w^{\prime}\langle b\rangle$ (which by definition necessarily terminates in a relative origin for $K^{\prime}$ ) has the form $w^{\prime}=w s$, where $w$ is a minimal (upward) geodesic stem for $K=w\langle b\rangle$. It follows that if two horocycles $K, \widetilde{K}$ share the same finite configuration type of relative radii over their uniformly bounded zones, then the collection of horocycles above $K$ will have the same configuration types (when restricted to their uniformly bounded zones) as the collection of horocycles above $\widetilde{K}$.

Let $\gamma=w v$ be a geodesic path representing the element $w b^{m}$ in $K=w\langle b\rangle$, where $w$ is a geodesic stem for $K$ and $v$ is a geodesic path for $b^{m}$. There is a corresponding path $\tilde{\gamma}=\tilde{w} v$ that represents $\tilde{w} b^{m}$ in $\widetilde{K}=\tilde{w}\langle b\rangle$, where the terminus of $\tilde{w}$ is the relative origin on $\widetilde{K}$ matching the terminus of $w$ on $K$. We are allowed to use $v$ as a suffix for $\tilde{\gamma}$ for two reasons. First, the horocycles above $\widetilde{K}$ exactly match those above $K$, at least when restricted to their respective uniformly bounded zones. Second, the cap of path $v$ will always lie strictly inside the bounded zone of the highest horocycle $v$ attains. This zone type is the same, whether the horocycle lies above $K$ or above $\widetilde{K}$. Now inductively add the letters of $v$ to each side of the cap of $v$ and in this way the entire path $v$ is constructed as a geodesic path connecting two vertices on $\widetilde{K}$.

We claim that $\tilde{\gamma}$ is geodesic (certainly, the prefix $\tilde{w}$ is geodesic). If not, a shorter representative for $\tilde{w} b^{m}$ must have the form $\hat{w} \hat{v}$, where $\hat{w}$ is a geodesic stem for $\widetilde{K}$ and $\hat{v}$ is a path connecting two vertices on $\widetilde{K}$ that is shorter than $v$. But in this case we can translate $\hat{v}$ to an isometric path connecting a relative origin of $K$ to $w b^{m}$. The concatenation of $\hat{v}$ onto a geodesic stem for this new relative origin yields a path for $w b^{m}$ that is shorter than the original $\gamma$, a contradiction.

Corollary 8.3. For any fixed $1<p<q$, there is a set of geodesic stems for all upper horocycles that forms a regular language. 
Proof. Let $\mathcal{U}$ be the set of all stems for upper horocycles that represent up-points. The collection $\mathcal{U}$ has an obvious groupoid structure under the operation of concatenation. Recall the definition of McCann cones from Section 5. Upward cones can be defined similarly. For an up-point represented by a geodesic stem $u$, the upward cone based at $u$ is the set of all up-point stem concatenations $u_{1} u_{2} \ldots u_{k}$, where $u u_{1} u_{2} \ldots u_{k}$ itself represents an up-point. The proof of Proposition 8.2 shows that $\mathcal{U}$ has only finitely many cone types $($ see $[\mathbf{5}, \mathbf{1 1}]$ ) and is therefore accepted by a (unique, minimal, deterministic) finite-state automaton.

We now generalize Proposition 6.2 to show that the Schofield geodesics form a combing for $b^{r}$, $r \gg 0$. We will ignore those small values of $r$ for which $b^{r}$ is itself geodesic. For fixed $1<p<q$, we assume the existence of finitely many upper horocycle equivalence classes, their up-points, and a minimal deterministic finite-state automaton that accepts (geodesic strings representing) all the up-points, as per Corollary 8.3. Adapting the remarks prior to Proposition 6.1, we delete any transitions that create duplicate paths for up-points. We also change to non-accept any state that does not correspond to the rightmost up-point on an upper horocycle (see discussion prior to Proposition 6.2). Now we need to add caps and various modifications to this DFA. We use caps of length $k p$ up to the maximum cap length shown in the McCann algorithm of Figure 4.1. It is possible that two distinct geodesic prefixes can represent the same word once various caps and suffixes are added (see Figure 8.2). This was remedied in the $p=2, q=3$ case (after Proposition 6.1 above) by an apparent ad hoc duplication of DFA states based on examination of figures. It is not immediately apparent that this can be done in general. Is it possible that there is an infinite regress of corrections to be made?

Lemma 8.4. Suppose that a DFA exists to accept/generate unique prefixes for a Schofield combing of $\left\{b^{r}: r \gg 0\right\} \subseteq \mathrm{BS}(p, q)$. Then the DFA can be modified so as to accept only valid prefix + cap combinations by making finitely many state additions/alterations to the automaton.

Proof. Consider two Schofield-type paths $\gamma_{1}, \gamma_{2}$ from the origin of the Cayley graph to vertex $b^{r}$ as shown in Figure 8.2. Both paths are assumed to have geodesic prefixes generated by the DFA but distinct caps. (They will share a vertical suffix and meet prior to $b^{r}$; this is irrelevant to our argument.) Without loss of generality, we may assume that both paths lie in the same upper half-sheet, which half-sheet is quasi-isometric to the hyperbolic plane. Thus, $\gamma_{1}, \gamma_{2}$ synchronously fellow travel (cf. [11]) and the relator area between them is uniformly thin (depending only on $p, q$ and not on $\gamma_{1}$ or $\gamma_{2}$ ). Such a relator consists of horobricks glued together. Since the diameter of the relator bordered by $\gamma_{1}$ and $\gamma_{2}$ is uniformly bounded and there are only finitely many possible caps, there is a uniform bound on the number of sideby-side horobricks glued horizontally. There are only finitely many possible ways to glue these side-by-side horobrick strips on top of each other, which implies lots of repeated configurations of these horobrick gluings. Successive up-points for $\gamma_{1}$ and $\gamma_{2}$ form the sides of these horobrick gluings and must be repeated when $\gamma_{1}$ and $\gamma_{2}$ are long. Therefore, we need only double check states in our automaton for duplicate string + cap combinations up to the bound on this finite configuration size.

The argument of Proposition 6.2 can be generalized for any fixed $1<p<q$. The adjustments made to our DFA via Lemma 8.4 ensure that geodesic stem + cap combinations are unique. To show existence, we again start with the combing $\overline{\mathcal{M}}$ of reversed McCann geodesics. For a given $r \gg 0$, there is a unique reversed McCann geodesic $\bar{w}$ representing $b^{r}$ having the form $\bar{w}=v c T^{k}$, where $v$ is (a geodesic stem for) an up-point, $c$ is a cap, and $k$ is the number of occurrences of $t$ in the string $v$. The DFA of Corollary 8.3 generates/accepts $v$. Either we accepted the prefix + cap combination $v c$ during the checking described in Lemma 8.4 (in which 


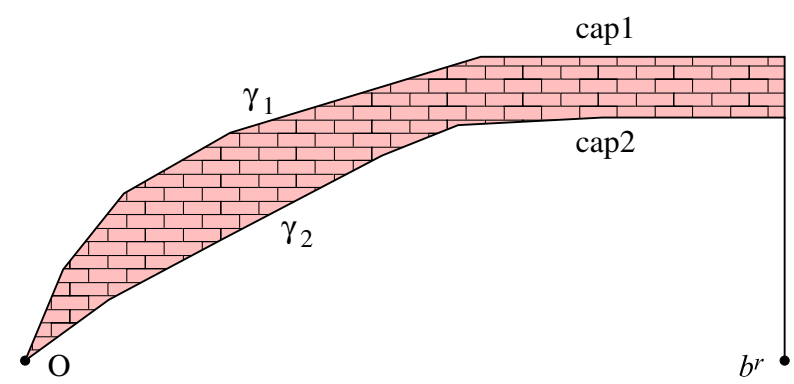

FiguRE 8.2. The paths $\gamma_{1}$ and $\gamma_{2}$ representing $b^{r}$ fellow travel and define a uniformly bounded relator.

case the argument is finished), or we discarded the combination $v c$ in favor of another prefix + cap combination $v^{\prime} c^{\prime}$, say. In the latter case, $b^{r}$ is represented by the Schofield geodesic $w^{\prime}=v^{\prime} c^{\prime} T^{j}$, where $j$ is the number of occurrences of $t$ in the string $v^{\prime}$. We have proved the following theorem.

Theorem 8.5. For fixed $1<p<q$, there is a unique Schofield geodesic representing $b^{r} \in \operatorname{BS}(p, q)$ for each $r \gg 0$.

TheOREm 8.6. When $p \nmid q$, the geodesic combing of the horocyclic subgroup by Schofield geodesics forms a context-sensitive language. Furthermore, the time complexity of acceptance is quadratic.

Proof. The Turing machine described for $\mathrm{BS}(2,3)$ can be modified for the general case $p \nmid q$. The general-case Turing machine uses several tapes and has several different caps for Schofield geodesics (we model this machine on the concrete example shown in [1]). We change the stem recognition states and create several cap states accordingly. The balancing of $t T$ pairs proceeds as in [1]. The cap is then replaced by a base $p$ number which represents the cap length and corresponding arithmetic steps are performed in base $p$ depending upon the symbols read. Although more scratch space for arithmetic operations may be needed on a third tape, the total number of tape cells necessary is bounded by a linear function of the length of the input word.

We count the number of tape-head moves required for acceptance in a worst-case scenario. We will use the standard 'big O' and 'big Theta' terminology. Observe as in [1] that for an input string of length $n \gg 0$ the time taken to reject an invalid word is less than the time taken to accept a Schofield geodesic word, because the Turing machine must complete the full procedure in the latter case but not in the former. Assume that $w$ is a Schofield geodesic of length $n$. Checking the regular language component of the prefix takes $\Theta(n)$ moves on the first tape. Determining the balance of $t, T$ pairs takes $\Theta\left(n^{2}\right)$ moves on the first tape, none on the second. The remaining binary arithmetic steps increment by +1 , decrement by -1 , or multiply by the constant $\frac{q}{p}$. Any one of these three operations can be done using $\mathcal{O}(n)$ tape moves on the second tape with one move on the first tape. We can also use $\mathcal{O}(n)$ moves on a third tape for help with the arithmetic (keeping track of base $p$ carries, etc). The total number of these individual arithmetic steps required is less than $n$; therefore, all the arithmetic can be done with $\mathcal{O}\left(n^{2}\right)$ moves. $\square$

The class of indexed languages are all context-sensitive but properly contain the treeadjoining languages. We do not know if the horocyclic subgroup admits an indexed combing by geodesics when $p \nmid q$. There is no known pumping lemma for indexed languages. Gilman’s shrinking lemma [16] gives necessary but insufficient criteria for a language to be indexed. The geodesic combings we have discussed easily satisfy the shrinking lemma test. 
The growing context-sensitive languages form a proper subclass of the context-sensitive languages [4]. It is also unknown if the Schofield geodesics form a growing context-sensitive language when $p \nmid q$. The observed irregularity of the geodesic cone types suggests that the growth series for the horocyclic subgroup in these cases is not rational or even $p$-recursive (that is, satisfying a linear recurrence with polynomial coefficients; synonyms are holonomic or d-finite). Numerical experimentation using Maple and Mathematica lends credence to these hypotheses, although no finite amount of data can disprove them. As of this printing, the Weingartner lemma and its corollaries are the only source of information we have that can be applied to more than finitely many terms of the growth series.

Conjecture 8.7. When $p \nmid q$, the growth series of the horocyclic subgroup is not $p$-recursive.

\section{Further applications when $p$ divides $q$}

Proposition 9.1. When $p \mid q$, the geodesic combing of the horocyclic subgroup by Schofield geodesics forms a deterministic one-counter, hence deterministic context-free, language.

Proof. Modify the Turing machine described for $\mathrm{BS}(p, q)$ as per Theorem 8.6 but, since $p \mid q$, no arithmetic is necessary. The geodesic stem is recognized by finitely many control states, while the balancing of $t T$ pairs requires a stack with only one alphabet symbol. Observe that the input word need be read only once from left to right without backtracking; the time complexity of recognition is linear.

Corollary 9.2. When $p \mid q$, the growth series of the horocyclic subgroup comprises a rational function.

Proof. Let us start with a fixed pair $p, q$ for which there is only a single cap associated to the Schofield combing of the horocyclic subgroup. Define a monoid homomorphism $\varphi$ : $\{b, B, t, T\}^{*} \rightarrow\{b, B, t, T\}^{*}$ by mapping $t$ to $t T$ and fixing the other letters. The image under $\varphi$ of the language of geodesic up-points is still a regular language. Concatenation of each string in this image with the cap $b^{k p}$ will still yield a regular language, with necessarily rational growth [11]. There is an obvious length-preserving bijection of the cap-adjoined $\varphi$-image with the Schofield geodesic combing (move all instances of $T$ to the end of each string), and the conclusion follows.

In the general case, there are several different caps $b^{p}, b^{2 p}, \ldots, b^{k p}$ for a specific Schofield combing. Consider the one-counter machine outlined in Proposition 9.1. It is built from the finite-state automaton whose states correspond to horocycle up-points (cf. Corollary 8.3) with each accept state leading to one or more cap string transitions. Suppose that $q$ is such an accept state which leads to distinct caps $b^{i p}, b^{j p}$; in other words, there are transitions labeled by geodesic stems $w_{i}, w_{j}$ which respectively lead into state $q$ in the machine such that $w_{i} b^{i p} T^{n}$ and $w_{j} b^{j p} T^{m}$ are accepted geodesics for some $n, m$. Make a duplicate $q_{i}$ of state $q$ but copy only the existing transitions into $q$ that can be coupled with the cap $b^{i p}$ and allow only $b^{i p}$ as the sole cap transition out of $q_{i}$ (but copy all other non-cap transitions out of $q_{i}$; cf. the details of [1, Proposition 3]). Similarly, make a duplicate $q_{j}$ of state $q$ but copy only the existing transitions into $q$ that can be coupled with the cap $b^{j p}$ and allow only $b^{j p}$ as the sole cap transition out of $q_{j}$ (but copy all other non-cap transitions out of $q_{j}$ ). Now erase the original state $q$ along with all of its in and out transitions.

The duplication process outlined in the preceding paragraph can be extended to original accept states with three or more cap assignments. After making all necessary duplications and erasures, we obtain a diagram with at most one cap assignment per state. Unfortunately, 
there may be multiple start states for this diagram if the original start state had several cap assignments. For a given cap $b^{l p}$, allow as accept states only those that have a valid $b^{l p}$ cap. Now we have the diagram of a one-counter machine for a Schofield-type language with a unique start state and a single cap. Its growth is rational by our original argument above. By varying this idea over all allowed caps, we see that the general case Schofield language is a disjoint union of finitely many languages with rational growth and the conclusion obtains.

Acknowledgements. We gratefully acknowledge the assistance of colleagues and students. Andreas Weingartner discovered the proof of Lemma 7.1. Jared Adams assisted with the details of Section 5. Based on a sketch given by the third author, Jared Adams and Chris McGahan solidified the Turing machine of Section 6 and wrote a working model [18] using the JFLAP software package written by Susan Rodger (www.jllap.org).

\section{Appendix}

The third author has compiled an exhaustive list of unambiguous context-free grammars that generate the Schofield geodesic combings in the cases when $p$ divides $q$. The method is based on the following idea: for a given class of pairs $(p, q)$, create a finite-state machine to generate the upward geodesic stems, append the appropriate mesa caps (with possible creation of new states/non-terminals), and balance each $t$ in the upward stem by a $T$ suffix. There is a correspondence between the states of the machine and the non-terminals of the grammar. Key words written in bold represent non-terminals; the italicized letters $b, B, t, T$ represent terminals. Note that unlike the explicitly worked example in Section 5 , these grammars generate the combings for all powers of $b$, both positive and negative.

The grammars can be converted to growth functions by the usual Chomsky-Schützenberger method (see for example [13]). We illustrate this again in the case of $\operatorname{BS}(n, 3 n), n>1$ but exclude proofs and most of the growth functions for the sake of brevity.

Grammar productions for the $\langle b\rangle$ subgroup of BS $(1,2)$

Start $\rightarrow$ Initial $\mid \mathbf{E}$

Initial $\rightarrow \epsilon|b| B\left|b^{2}\right| B^{2}$

$\mathbf{E} \rightarrow t \mathbf{E} T\left|b t^{2} \mathbf{E} T^{2}\right| B t^{2} \mathbf{E} T^{2} \mid \mathbf{C}$

$\mathbf{C} \rightarrow b^{3}\left|b^{4}\right| b^{5}\left|b t b^{3} T\right| b t b^{4} T\left|B^{3}\right| B^{4}\left|B^{5}\right| B t B^{3} T \mid B t B^{4} T$

Grammar productions for the $\langle b\rangle$ subgroup of $\mathrm{BS}(1,4)$

Start $\rightarrow \epsilon \mid \mathbf{C A P}$

CONJ $\rightarrow t$ CAP $T \mid b t$ FRONT $T$

$\mathbf{C O N J} \rightarrow\left|b^{2} t \mathbf{C A P}_{\mathbf{R}} T\right| B^{2} t \mathbf{C A P}_{\mathbf{L}} T \mid B t \mathbf{B A C K} T$

$\mathbf{C A P} \rightarrow \mathbf{C O N J}|b| B\left|b^{j}\right| B^{j}$

$\mathbf{F R O N T} \rightarrow \mathbf{C O N J}|b| b^{j} \mid B^{j}$

$\mathbf{B A C K} \rightarrow \mathbf{C O N J}|B| b^{j} \mid B^{j}$

$\mathbf{C A P}_{\mathbf{R}} \rightarrow t \mathbf{C A P} T|b t \mathbf{F R O N T} T| b^{H}$

$\mathbf{C A P}_{\mathbf{L}} \rightarrow t \mathbf{C A P} T|B t \mathbf{B A C K} T| B^{H}$

for $j \in\{2,3\} \quad H \in\{1,2\}$ 
Grammar productions for the $\langle b\rangle$ subgroup of $\mathrm{BS}(1,3)$

Start $\rightarrow$ INITIAL $\mid$ OFFSET $\mid t$ CAP $T$

INITIAL $\rightarrow \epsilon\left|b^{l}\right| B^{l} \quad$ for $l \in\{1,2\}$

OFFSET $\rightarrow B t$ BACK $T \mid b t$ FRONT $T$

CONJ $\rightarrow t$ CAP $T \mid b t$ FRONT $T \mid B t$ BACK $T$

$\mathbf{C A P} \rightarrow \mathbf{C O N J}|b| B\left|b^{2}\right| B^{2}$

$\mathbf{F R O N T} \rightarrow \mathbf{C O N J}|b| b^{2} \mid B^{2}$

$\mathbf{B A C K} \rightarrow \mathbf{C O N J}|B| b^{2} \mid B^{2}$

Grammar productions for the $\langle b\rangle$ subgroup of BS(1, $(2 m+1)), m>1$

Start $\rightarrow$ INITIAL $\mid$ OFFSET $\mid t$ CAP $T \mid b^{k} t$ CAP $T \mid B^{k} t$ CAP $T$

INITIAL $\rightarrow \epsilon\left|b^{l}\right| B^{l} \quad$ for $l \in\{1,2,3, \ldots,(m+1)\}$

OFFSET $\rightarrow B^{m} t$ BACK $T \mid b^{m} t$ FRONT $T$

$\mathbf{C O N J} \rightarrow t \mathbf{C A P} T\left|b^{k} t \mathbf{C A P} T\right| B^{k} t \mathbf{C A P} T$

CONJ $\rightarrow b^{m} t$ FRONT $T \mid B^{m} t$ BACK $T$

$\mathbf{C A P} \rightarrow \mathbf{C O N J}|b| B\left|b^{j}\right| B^{j}$

FRONT $\rightarrow$ CONJ $|b| b^{j} \mid B^{j}$

$\mathbf{B A C K} \rightarrow \mathbf{C O N J}|B| b^{j} \mid B^{j}$

where $j \in\{2,3, \ldots, m+1\}, \quad k \in\{1,2,3, \ldots, m-1\}, \quad$ and $\quad r \in\{1,2,3, \ldots, m\}$

Grammar productions for the $\langle b\rangle$ subgroup of $\mathrm{BS}(1,2 m), m \geqslant 3$

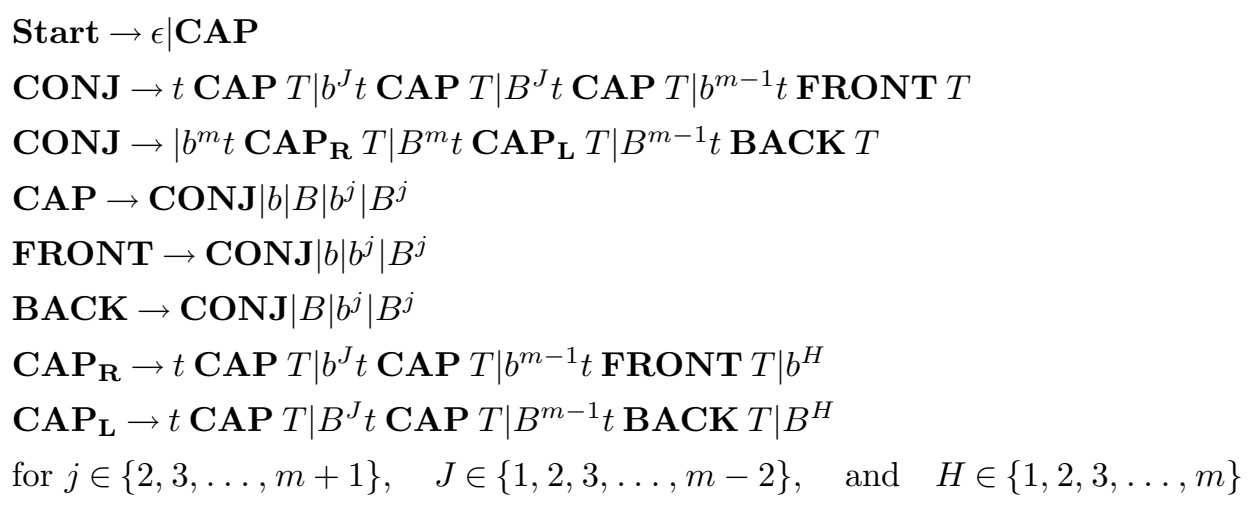


Grammar for the $\langle b\rangle$ subgroup of $\mathrm{BS}(2,4)$

Start $\rightarrow \epsilon\left|b^{i}\right| B^{i} \mid$ CONJ $\mid b t$ FRONT $T \mid B t$ BACK $T$

$\mathbf{C O N J} \rightarrow t \mathbf{C A P} T\left|b^{2} t \mathbf{C A P}_{\mathbf{R}} T\right| B^{2} t \mathbf{C A P}_{\mathbf{L}} T$

$\mathbf{C A P} \rightarrow \mathbf{C O N J}\left|b^{2}\right| B^{2}$

FRONT $\rightarrow$ CONJ $\mid b^{2}$

$\mathrm{BACK} \rightarrow \mathrm{CONJ} \mid B^{2}$

$\mathbf{C A P}_{\mathbf{R}} \rightarrow t$ FRONT $T \mid b^{2}$

$\mathbf{C A P}_{\mathbf{L}} \rightarrow t \mathbf{B A C K} T \mid B^{2}$

for $i \in\{1,2,3\}$

Grammar for the $\langle b\rangle$ subgroup of $\mathrm{BS}(2,8)$

Start $\rightarrow \epsilon\left|b^{i}\right| B^{i} \mid b^{3} t$ FRONT $T \mid B^{3} t$ BACK $T \mid$ CONJ

Start $\rightarrow b t$ CAP $T \mid B t$ CAP $T$

$\mathbf{C O N J} \rightarrow t \mathbf{C A P} T\left|b^{2} t \mathbf{C A P} T\right| B^{2} t \mathbf{C A P} T \mid b^{4} t \mathbf{C A P}_{\mathbf{R}} T$

$\mathbf{C O N J} \rightarrow B^{4} t \mathbf{C A P}_{\mathbf{L}} T$

$\mathbf{C A P} \rightarrow \mathbf{C O N J}\left|b^{2}\right| B^{2}\left|b^{4}\right| B^{4}$

FRONT $\rightarrow$ CONJ $\left|b^{2}\right| b^{4} \mid B^{4}$

$\mathrm{BACK} \rightarrow \mathbf{C O N J}\left|B^{2}\right| b^{4} \mid B^{4}$

$\mathbf{C A P}_{\mathbf{R}} \rightarrow t \mathbf{C A P} T\left|b^{2} t \mathbf{F R O N T} T\right| b^{2 H}$

$\mathbf{C A P}_{\mathbf{L}} \rightarrow t \mathbf{C A P} T\left|B^{2} t \mathbf{B A C K} T\right| B^{2 H}$

for $H \in\{1,2\} \quad$ and $\quad i \in\{1,2,3,4,5\}$

Grammar for the $\langle b\rangle$ subgroup of $\operatorname{BS}(2,4 m)$, where $m \geq 3$

Start $\rightarrow \epsilon\left|b^{i}\right| B^{i} \mid t$ CAP $T \mid b^{J} t$ CAP $T \mid B^{J} t$ CAP $T$

Start $\rightarrow b^{2 m-1} t$ FRONT $T \mid B^{2 m-1} t$ BACK $T$

Start $\rightarrow b^{2 m} t \mathbf{C A P}_{\mathbf{R}} T \mid B^{2 m} t \mathbf{C A P}_{\mathbf{L}} T$

$\mathbf{C O N J} \rightarrow t \mathbf{C A P} T\left|b^{2 j} t \mathbf{C A P} T\right| B^{2 j} t \mathbf{C A P} T \mid b^{2 m} t \mathbf{C A P}_{\mathbf{R}} T$

$\mathbf{C O N J} \rightarrow B^{2 m} t \mathbf{C A P}_{\mathbf{L}} T$

$\mathbf{C A P} \rightarrow \mathbf{C O N J}\left|b^{2}\right| B^{2}\left|b^{2 j+2}\right| B^{2 j+2}$

FRONT $\rightarrow$ CONJ $\left|b^{2}\right| b^{2 j+2} \mid B^{2 j+2}$

$\mathbf{B A C K} \rightarrow \mathbf{C O N J}\left|B^{2}\right| b^{2 j+2} \mid B^{2 j+2}$

$\mathbf{C A P}_{\mathbf{R}} \rightarrow t \mathbf{C A P} T\left|b^{2 h} t \mathbf{C A P} T\right| b^{2(m-1)} t$ FRONT $T \mid b^{2 H}$

$\mathbf{C A P}_{\mathbf{L}} \rightarrow t \mathbf{C A P} T\left|B^{2 h} t \mathbf{C A P} T\right| B^{2(m-1)} t$ BACK $T \mid B^{2 H}$

for $J \in\{1,2,3, \ldots, 2 m-2\}, \quad j \in\{1,2,3, \ldots, m-1\}$,

$H \in\{1,2,3, \ldots, m\}, \quad h \in\{1,2,3, \ldots, m-2\}, \quad$ and $\quad i \in\{1,2,3, \ldots, 2 m+1\}$ 
Grammar for the $\langle b\rangle$ subgroup of $\mathrm{BS}(3,6)$

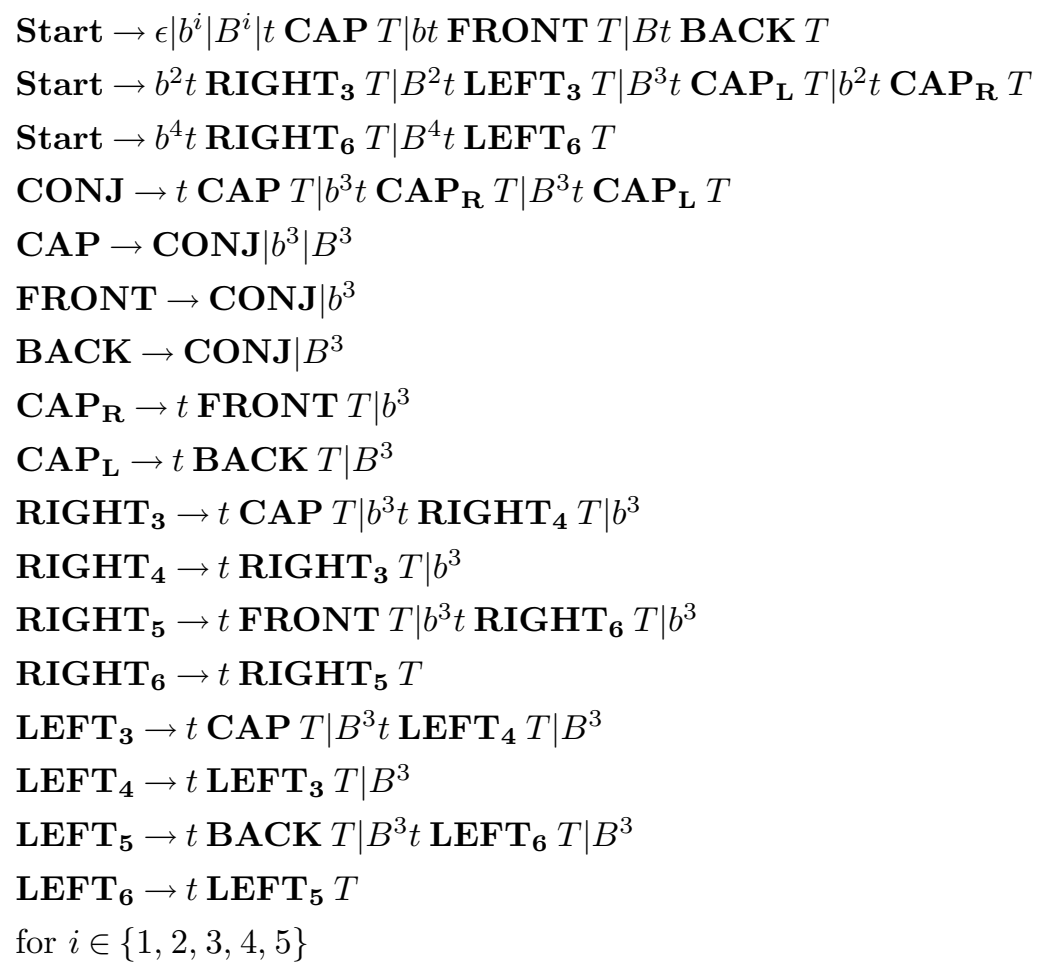

Grammar for the $\langle b\rangle$ subgroup of $\mathrm{BS}(3,12)$

Start $\rightarrow \epsilon\left|b^{i}\right| B^{i}|t \mathbf{C A P} T| b^{h} t \mathbf{C A P} T \mid B^{h} t \mathbf{C A P} T$

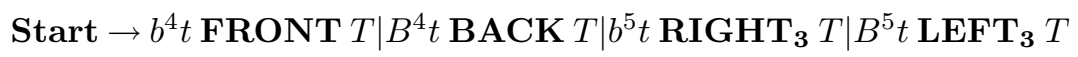

Start $\rightarrow B^{6} t \mathbf{C A P}_{\mathbf{L}} T\left|b^{6} t \mathbf{C A P}_{\mathbf{R}} T\right| b^{7} t \mathbf{R I G H T}_{\mathbf{6}} T \mid B^{7} t \mathbf{L E F T}_{\mathbf{6}} T$

$\mathbf{C O N J} \rightarrow t \mathbf{C A P} T\left|b^{3} t \mathbf{C A P} T\right| B^{3} t \mathbf{C A P} T\left|b^{6} t \mathbf{C A P}_{\mathbf{R}} T\right| B^{6} t \mathbf{C A P}_{\mathbf{L}} T$

$\mathbf{C A P} \rightarrow \mathbf{C O N J}\left|b^{3}\right| B^{3}\left|b^{6}\right| B^{6}$

$\mathbf{F R O N T} \rightarrow \mathbf{C O N J}\left|b^{3}\right| b^{6} \mid B^{6}$

$\mathbf{B A C K} \rightarrow \mathbf{C O N J}\left|B^{3}\right| b^{6} \mid B^{6}$

$\mathbf{C A P}_{\mathbf{R}} \rightarrow t \mathbf{C A P} T \mid b^{3} t$ FRONT $T \mid b^{3 H}$

$\mathbf{C A P}_{\mathbf{L}} \rightarrow t \mathbf{C A P} T \mid B^{3} t$ BACK $T \mid B^{3 H}$

RIGHT $_{\mathbf{3}} \rightarrow t \mathbf{C A P} T \mid b^{3} t$ CAP $T \mid b^{6} t$ RIGHT $_{\mathbf{4}} T \mid b^{3 H}$

$\mathbf{R I G H T}_{\mathbf{4}} \rightarrow t \mathbf{C A P} T\left|b^{3} t \mathbf{R I G H T}_{\mathbf{3}} T\right| b^{3 H}$

$\mathbf{R I G H T}_{\mathbf{5}} \rightarrow t \mathbf{C A P} T \mid b^{3} t$ FRONT $T \mid b^{6} t$ RIGHT $_{\mathbf{6}} T \mid b^{3 H}$

$\mathbf{R I G H T}_{\mathbf{6}} \rightarrow t \mathbf{C A P} T\left|b^{3} t \mathbf{R I G H T}_{\mathbf{5}} T\right| b^{3}$

$\mathbf{L E F T}_{\mathbf{3}} \rightarrow t$ CAP $T \mid B^{3} t$ CAP $T\left|B^{6} t \mathbf{L E F T}_{\mathbf{4}} T\right| B^{3 H}$

$\mathbf{L E F T}_{\mathbf{4}} \rightarrow t$ CAP $T\left|B^{3} t \mathbf{L E F T}_{\mathbf{3}} T\right| B^{3 H}$

$\mathbf{L E F T}_{\mathbf{5}} \rightarrow t \mathbf{C A P} T\left|B^{3} t \mathbf{B A C K} T\right| B^{6} t \mathbf{L E F T}_{\mathbf{6}} T \mid B^{3 H}$

$\mathbf{L E F T}_{\mathbf{6}} \rightarrow t \mathbf{C A P} T\left|B^{3} t \mathbf{L E F T}_{\mathbf{5}} T\right| B^{3}$

for $h \in\{1,2,3\}, \quad H \in\{1,2\}, \quad$ and $\quad i \in\{1,2,3,4,5,6,7,8\}$ 
Grammar for the $\langle b\rangle$ subgroup of $\mathrm{BS}(3,6 m)$, assuming $m>2$

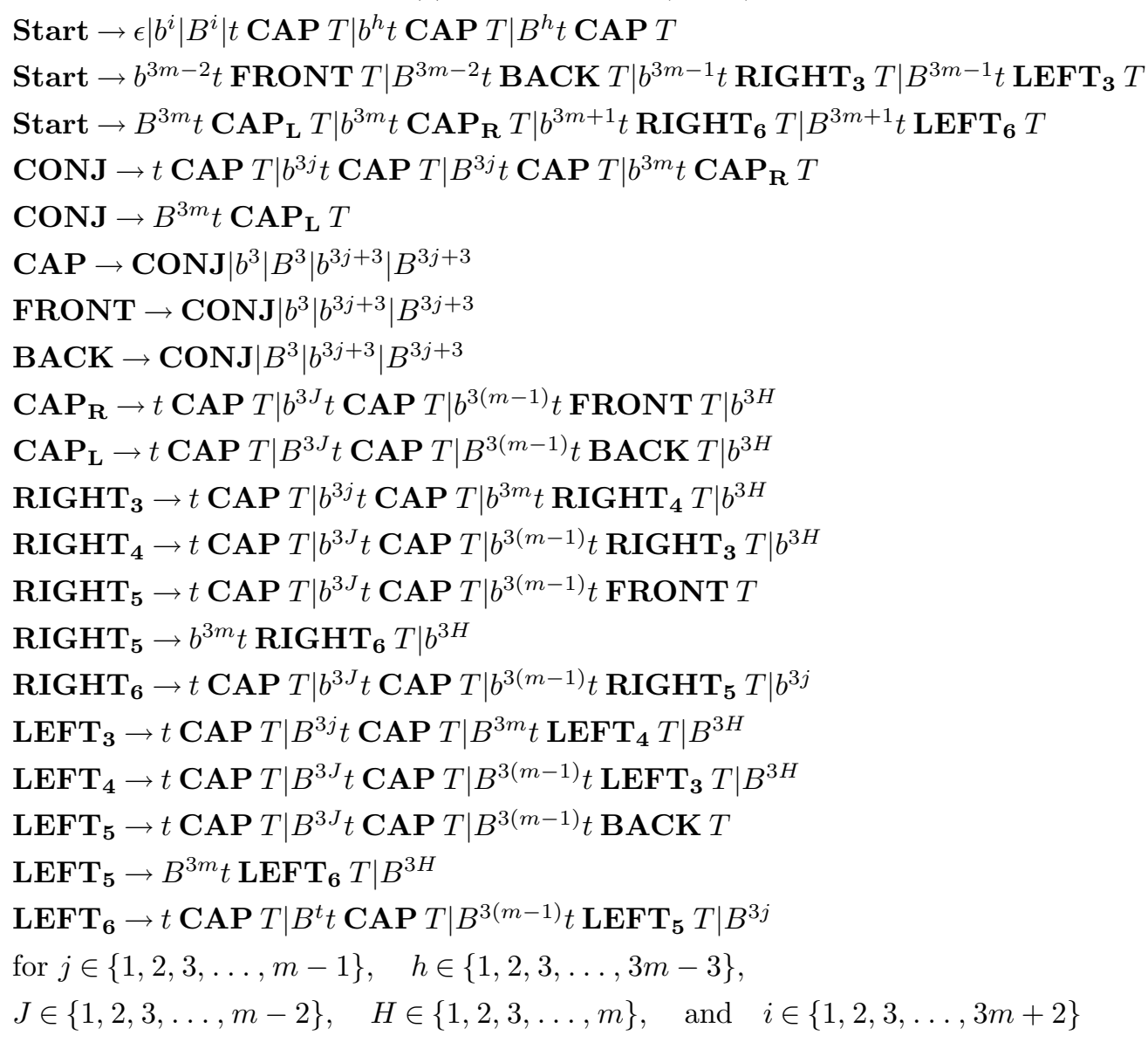

Grammar for the $\langle b\rangle$ subgroup of $\operatorname{BS}(n, 2 n)$, where $n=2 k$ and $k>1$

Start $\rightarrow \epsilon\left|b^{i}\right| B^{i}|t \mathbf{C A P} T| b^{L} t \mathbf{C A P} T \mid B^{L} t \mathbf{C A P} T$

Start $\rightarrow b^{k} t$ FRONT $T \mid B^{k} t$ BACK $T\left|b^{n} t \mathbf{C A P}_{\mathbf{R}} T\right| B^{n} t \mathbf{C A P}_{\mathbf{L}} T$

Start $\rightarrow b^{n-L} t$ RIGHT $_{\mathbf{1}} T \mid B^{n-L} t$ LEFT $_{\mathbf{1}} T \mid B^{n+L} t$ LEFT $T \mid b^{n+L} t$ RIGHT $T$

$\mathbf{C O N J} \rightarrow t \mathbf{C A P} T\left|b^{n} t \mathbf{C A P}_{\mathbf{R}} T\right| B^{n} t \mathbf{C A P}_{\mathbf{L}} T$

$\mathbf{C A P} \rightarrow \mathbf{C O N J}\left|b^{n}\right| B^{n}$

FRONT $\rightarrow$ CONJ $\mid b^{n}$

$\mathrm{BACK} \rightarrow \mathrm{CONJ} \mid B^{n}$

$\mathbf{C A P}_{\mathbf{R}} \rightarrow t \mathbf{F R O N T} T \mid b^{n}$

$\mathbf{C A P}_{\mathbf{L}} \rightarrow t \mathbf{B A C K} T \mid B^{n}$

RIGHT $\rightarrow t$ RIGHT $_{\mathbf{1}} T$

$\mathbf{L E F T} \rightarrow t$ LEFT $_{\mathbf{1}} T$

RIGHT $_{\mathbf{1}} \rightarrow t$ CAP $T \mid b^{n} t$ RIGHT $T \mid b^{n}$

LEFT $_{\mathbf{1}} \rightarrow t$ CAP $T \mid B^{n} t$ LEFT $T \mid B^{n}$

for $L \in\{1,2,3, \ldots, k-1\} \quad$ and $\quad i \in\{1,2,3, \ldots, n+k\}$ 
Grammar for the $\langle b\rangle$ subgroup of $\operatorname{BS}(n, 2 n)$, assuming $n=2 k+1$ and $k>1$

Start $\rightarrow \epsilon\left|b^{i}\right| B^{i}|t \mathbf{C A P} T| b^{h} t \mathbf{C A P} T \mid B^{h} t \mathbf{C A P} T$

Start $\rightarrow b^{n-k-1} t$ FRONT $T \mid B^{n-k-1} t$ BACK $T \mid b^{n-k} t$ RIGHT $_{\mathbf{3}} T \mid B^{n-k} t$ LEFT $_{\mathbf{3}} T$

Start $\rightarrow B^{n-L} t \mathbf{L E F T}_{\mathbf{1}} T\left|b^{n-L} t \mathbf{R I G H T}_{\mathbf{1}} T\right| B^{n} t \mathbf{C A P}_{\mathbf{L}} T \mid b^{n} t \mathbf{C A P}_{\mathbf{R}} T$

Start $\rightarrow B^{n+L} t$ LEFT $_{\mathbf{2}} T \mid b^{n+L} t$ RIGHT $_{\mathbf{2}} T \mid b^{n+k} t$ RIGHT $_{\mathbf{6}} T \mid B^{n+k} t \mathbf{L E F T}_{\mathbf{6}} T$

$\mathbf{C O N J} \rightarrow t \mathbf{C A P} T\left|b^{n} t \mathbf{C A P}_{\mathbf{R}} T\right| B^{n} t \mathbf{C A P}_{\mathbf{L}} T$

$\mathbf{C A P} \rightarrow \mathbf{C O N J}\left|b^{n}\right| B^{n}$

FRONT $\rightarrow$ CONJ $\mid b^{n}$

$\mathrm{BACK} \rightarrow \mathrm{CONJ} \mid B^{n}$

$\mathbf{C A P}_{\mathbf{R}} \rightarrow t$ FRONT $T \mid b^{n}$

$\mathbf{C A P}_{\mathbf{L}} \rightarrow t \mathbf{B A C K} T \mid B^{n}$

RIGHT $_{\mathbf{1}} \rightarrow t$ CAP $T \mid b^{n} t$ RIGHT $_{\mathbf{2}} T \mid b^{n}$

RIGHT $_{\mathbf{2}} \rightarrow t$ RIGHT $_{\mathbf{1}} T$

RIGHT $_{\mathbf{3}} \rightarrow t$ CAP $T \mid b^{n} t$ RIGHT $_{\mathbf{4}} T \mid b^{n}$

RIGHT $_{\mathbf{4}} \rightarrow t$ RIGHT $_{\mathbf{3}} T \mid b^{n}$

$\mathbf{R I G H T}_{\mathbf{5}} \rightarrow t$ FRONT $T \mid b^{n} t$ RIGHT $_{\mathbf{6}} T \mid b^{n}$

RIGHT $_{\mathbf{6}} \rightarrow t$ RIGHT $_{\mathbf{5}} T$

$\mathbf{L E F T}_{\mathbf{1}} \rightarrow t$ CAP $T\left|B^{n} t \mathbf{L E F T}_{\mathbf{2}} T\right| B^{n}$

$\mathbf{L E F T}_{\mathbf{2}} \rightarrow t \mathbf{L E F T}_{\mathbf{1}} T$

$\mathbf{L E F T}_{\mathbf{3}} \rightarrow t$ CAP $T\left|B^{n} t \mathbf{L E F T}_{\mathbf{4}} T\right| B^{n}$

$\mathbf{L E F T}_{\mathbf{4}} \rightarrow t \mathbf{L E F T}_{\mathbf{3}} T \mid B^{n}$

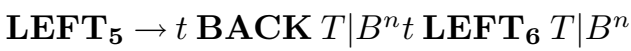

$\mathbf{L E F T}_{\mathbf{6}} \rightarrow t \mathbf{L E F T}_{\mathbf{5}} T$

for $h \in\{1,2,3, \ldots, n-k-2\}, \quad L \in\{1,2,3, \ldots, k-1\}, \quad$ and $\quad i \in\{1,2,3, \ldots, n+k+1\}$ 
Grammar for a context-free geodesic combing of the horocyclic subgroup of $\mathrm{BS}(n, 3 n)$ with $n>1$

Start $\rightarrow$ INIT $\mid$ OFFSET $\mid t$ CAP $T \mid b^{i} t$ CAP $T \mid B^{i} t$ CAP $T$

INIT $\rightarrow \epsilon\left|b^{l}\right| B^{l}$

OFFSET $\rightarrow b^{n+i} t$ RIGHT $T \mid B^{n+i} t$ LEFT $T \mid B^{n} t$ BACK $T \mid b^{n} t$ FRONT $T$

CONJ $\rightarrow t$ CAP $T \mid b^{n} t$ FRONT $T \mid B^{n} t$ BACK $T$

$\mathbf{C A P} \rightarrow \mathbf{C O N J}\left|b^{n}\right| B^{n}\left|b^{2 n}\right| B^{2 n}$

FRONT $\rightarrow$ CONJ $\left|b^{n}\right| b^{2 n} \mid B^{2 n}$

$\mathbf{B A C K} \rightarrow \mathbf{C O N J}\left|B^{n}\right| b^{2 n} \mid B^{2 n}$

RIGHT $\rightarrow t$ CAP $T \mid b^{n} t$ RIGHT $T \mid b^{n}$

$\mathbf{L E F T} \rightarrow t \mathbf{C A P} T\left|B^{n} t \mathbf{L E F T} T\right| B^{n}$

where $i \in\{1,2,3, \ldots, n-1\} \quad$ and $\quad l \in\{1,2,3, \ldots, 2 n\}$

Chomsky-Schützenberger equations for the previous grammar: replace each production arrow with equality, all terminals become the variable $z$, '|' becomes ' + ', and juxtaposition becomes (commutative) multiplication.

Start $=\mathbf{I N I T}+$ OFFSET $+z^{2} \mathbf{C A P}+2 \mathbf{C A P}\left(\sum_{i=1}^{n-1} z^{i+2}\right)$.

$\mathbf{I N I T}=1+2\left(\sum_{l=1}^{2 n} z^{l}\right)$

OFFSET $=z^{n+2}($ FRONT + BACK $)+\left(\sum_{i=1}^{n-1} z^{n+i+2}\right)($ LEFT + RIGHT $)$.

$\mathbf{C O N J}=z^{2} \mathbf{C A P}+z^{n+2}(\mathbf{F R O N T}+\mathbf{B A C K})$

$\mathbf{C A P}=\mathbf{C O N J}+2 z^{n}+2 z^{2 n}$

FRONT $=$ CONJ $+z^{n}+2 z^{2 n}$

$\mathrm{BACK}=$ FRONT

RIGHT $=z^{2} \mathbf{C A P}+z^{n+2} \mathbf{R I G H T}+z^{n}$

LEFT $=$ RIGHT

Solving this system of equations for Start yields the spherical growth function:

$$
\frac{(1+z)\left(1+z-z^{2+n}-z^{3+n}-2 z^{1+2 n}+2 z^{3+2 n}-2 z^{2+3 n}+2 z^{3+3 n}\right)}{1-z^{2}-3 z^{2+n}+z^{4+n}+2 z^{4+2 n}}
$$


Grammar for the $\langle b\rangle$ subgroup of $\operatorname{BS}(n, 4 n)$, assuming $n=2 k+1$ and $k>1$

Start $\rightarrow \epsilon\left|b^{i}\right| B^{i}|t \mathbf{C A P} T| b^{h} t \mathbf{C A P} T \mid B^{h} t$ CAP $T$

Start $\rightarrow b^{2 n-k-1} t$ FRONT $T \mid B^{2 n-k-1} t$ BACK $T$

Start $\rightarrow b^{2 n-k} t$ RIGHT $_{\mathbf{3}} T \mid B^{2 n-k} t \mathbf{L E F T}_{\mathbf{3}} T$

Start $\rightarrow B^{2 n-L} t \mathbf{L E F T}_{\mathbf{1}} \mid b^{2 n-L} t \mathbf{R I G H T}_{\mathbf{1}} T$

Start $\rightarrow B^{2 n} t \mathbf{C A P}_{\mathbf{L}} T \mid b^{2 n} t \mathbf{C A P}_{\mathbf{R}} T$

Start $\rightarrow B^{2 n+L} t$ LEFT $_{\mathbf{2}} T \mid b^{2 n+L} t$ RIGHT $_{\mathbf{2}} T$

Start $\rightarrow b^{2 n+k} t \mathbf{R I G H T}_{\mathbf{6}} T \mid B^{2 n+k} t \mathbf{L E F T}_{\mathbf{6}} T$

$\mathbf{C O N J} \rightarrow t \mathbf{C A P} T\left|b^{n} t \mathbf{C A P} T\right| B^{n} t \mathbf{C A P} T \mid b^{2 n} t \mathbf{C A P}_{\mathbf{R}} T$

$\mathbf{C O N J} \rightarrow B^{2 n} t \mathbf{C A P}_{\mathbf{L}} T$

$\mathbf{C A P} \rightarrow \mathbf{C O N J}\left|b^{n}\right| B^{n}\left|b^{2 n}\right| B^{2 n}$

$\mathbf{F R O N T} \rightarrow \mathbf{C O N J}\left|b^{n}\right| b^{2 n} \mid B^{2 n}$

$\mathbf{B A C K} \rightarrow \mathbf{C O N J}\left|B^{n}\right| b^{2 n} \mid B^{2 n}$

$\mathbf{C A P}_{\mathbf{R}} \rightarrow t \mathbf{C A P} T\left|b^{n} t \mathbf{F R O N T} T\right| b^{H n}$

$\mathbf{C A P}_{\mathbf{L}} \rightarrow t \mathbf{C A P} T\left|B^{n} t \mathbf{B A C K} T\right| B^{H n}$

$\mathbf{R I G H T}_{\mathbf{1}} \rightarrow t \mathbf{C A P} T\left|b^{n} t \mathbf{C A P} T\right| b^{2 n} t \mathbf{R I G H T}_{\mathbf{2}} T \mid b^{H n}$

$\mathbf{R I G H T}_{\mathbf{2}} \rightarrow t \mathbf{C A P} T\left|b^{n} t \mathbf{R I G H T}_{\mathbf{1}} T\right| b^{n}$

$\mathbf{R I G H T}_{\mathbf{3}} \rightarrow t \mathbf{C A P} T\left|b^{n} t \mathbf{C A P} T\right| b^{2 n} t \mathbf{R I G H T}_{\mathbf{4}} T \mid b^{H n}$

$\mathbf{R I G H T}_{\mathbf{4}} \rightarrow t \mathbf{C A P} T\left|b^{n} t \mathbf{R I G H T}_{\mathbf{3}} T\right| b^{H n}$

$\mathbf{R I G H T}_{\mathbf{5}} \rightarrow t \mathbf{C A P} T\left|b^{n} t \mathbf{F R O N T} T\right| b^{2 n} t \mathbf{R I G H T}_{\mathbf{6}} T \mid b^{H n}$

$\mathbf{R I G H T}_{\mathbf{6}} \rightarrow t \mathbf{C A P} T\left|b^{n} t \mathbf{R I G H T}_{\mathbf{5}} T\right| b^{n}$

$\mathbf{L E F T}_{\mathbf{1}} \rightarrow t$ CAP $T \mid B^{n} t$ CAP $T\left|B^{2 n} t \mathbf{L E F T}_{\mathbf{2}} T\right| B^{H n}$

$\mathbf{L E F T}_{\mathbf{2}} \rightarrow t \mathbf{C A P} T\left|B^{n} t \mathbf{L E F T}_{\mathbf{1}} T\right| B^{n}$

$\mathbf{L E F T}_{\mathbf{3}} \rightarrow t \mathbf{C A P} T\left|B^{n} t \mathbf{C A P} T\right| B^{2 n} t \mathbf{L E F T}_{\mathbf{4}} T \mid B^{H n}$

$\mathbf{L E F T}_{\mathbf{4}} \rightarrow t \mathbf{C A P} T\left|B^{n} t \mathbf{L E F T}_{\mathbf{3}} T\right| B^{H n}$

$\mathbf{L E F T}_{\mathbf{5}} \rightarrow t \mathbf{C A P} T\left|B^{n} t \mathbf{B A C K} T\right| B^{2 n} t \mathbf{L E F T}_{\mathbf{6}} T \mid B^{H n}$

$\mathbf{L E F T}_{\mathbf{6}} \rightarrow t \mathbf{C A P} T\left|B^{n} t \mathbf{L E F T}_{\mathbf{5}} T\right| B^{n}$

for $h \in\{1,2,3, \ldots, 2 n-k-2\}, \quad H \in\{1,2\}, \quad L \in\{1,2,3, \ldots, k-1\}$,

and $i \in\{1,2,3, \ldots, 2 n+k+1\}$ 
Grammar for the $\langle b\rangle$ subgroup of $\operatorname{BS}(n, 4 n)$, where $n=2 k$ and $n>1$

Start $\rightarrow \epsilon\left|b^{i}\right| B^{i}|t \mathbf{C A P} T| b^{h} t \mathbf{C A P} T \mid B^{h} t \mathbf{C A P} T$

Start $\rightarrow b^{2 n-k} t$ FRONT $T \mid B^{2 n-k} t$ BACK $T$

Start $\rightarrow b^{2 n} t \mathbf{C A P}_{\mathbf{R}} T \mid B^{2 n} t \mathbf{C A P}_{\mathbf{L}} T$

Start $\rightarrow b^{2 n-L} t$ RIGHT $_{\mathbf{1}} T \mid B^{2 n-L} t$ LEFT $_{\mathbf{1}} T$

Start $\rightarrow B^{2 n+L} t$ LEFT $T \mid b^{2 n+L} t$ RIGHT $T$

$\mathbf{C O N J} \rightarrow t \mathbf{C A P} T\left|b^{n} t \mathbf{C A P} T\right| B^{n} t \mathbf{C A P} T \mid b^{2 n} t \mathbf{C A P}_{\mathbf{R}} T$

$\mathbf{C O N J} \rightarrow B^{2 n} t \mathbf{C A P}_{\mathbf{L}} T$

$\mathbf{C A P} \rightarrow \mathbf{C O N J}\left|b^{n}\right| B^{n}\left|b^{2 n}\right| B^{2 n}$

FRONT $\rightarrow$ CONJ $\left|b^{n}\right| b^{2 n} \mid B^{2 n}$

$\mathrm{BACK} \rightarrow \mathbf{C O N J}\left|B^{n}\right| b^{2 n} \mid B^{2 n}$

$\mathbf{C A P}_{\mathbf{R}} \rightarrow t \mathbf{C A P} T\left|b^{n} t \mathbf{F R O N T} T\right| b^{H n}$

$\mathbf{C A P}_{\mathbf{L}} \rightarrow t \mathbf{C A P} T\left|B^{n} t \mathbf{B A C K} T\right| B^{H n}$

RIGHT $\rightarrow t$ CAP $T \mid b^{n} t$ RIGHT $_{\mathbf{1}} T \mid b^{n}$

$\mathbf{L E F T} \rightarrow t$ CAP $T\left|B^{n} t \mathbf{L E F T}_{\mathbf{1}} T\right| B^{n}$

RIGHT $_{\mathbf{1}} \rightarrow t$ CAP $T \mid b^{n} t$ CAP $T \mid b^{2 n} t$ RIGHT $T \mid b^{H n}$

$\mathbf{L E F T}_{\mathbf{1}} \rightarrow t$ CAP $T \mid B^{n} t$ CAP $T \mid B^{2 n} t$ LEFT $T \mid B^{H n}$

for $H \in\{1,2\}, \quad h \in\{1,2,3,4, \ldots, 2 n-k-1\}, \quad L \in\{1,2,3, \ldots, k-1\}$,

and $i \in\{1,2,3, \ldots, 2 n+k\}$

Grammar for the $\langle b\rangle$ subgroup of $\operatorname{BS}(n,(2 m+1) n)$ for $n>1$ and $m>1$

Start $\rightarrow$ INITIAL $\mid$ OFFSET $\mid t$ CAP $T \mid b^{h} t$ CAP $T \mid B^{h} t$ CAP $T$

INITIAL $\rightarrow \epsilon\left|b^{l}\right| B^{l} \quad$ for $l \in\{1,2,3, \ldots, n(m+1)\}$

OFFSET $\rightarrow b^{m n+i} t$ RIGHT $T \mid B^{n m+i} t$ LEFT $T$

OFFSET $\rightarrow B^{n m} t$ BACK $T \mid b^{m n} t$ FRONT $T$

$\mathbf{C O N J} \rightarrow t \mathbf{C A P} T\left|b^{k n} t \mathbf{C A P} T\right| B^{k n} t$ CAP $T$

CONJ $\rightarrow b^{m n} t$ FRONT $T \mid B^{n m} t$ BACK $T$

$\mathbf{C A P} \rightarrow \mathbf{C O N J}\left|b^{n}\right| B^{n}\left|b^{j n}\right| B^{j n}$

$\mathbf{F R O N T} \rightarrow \mathbf{C O N J}\left|b^{n}\right| b^{j n} \mid B^{j n}$

$\mathbf{B A C K} \rightarrow \mathbf{C O N J}\left|B^{n}\right| b^{j n} \mid B^{j n}$

RIGHT $\rightarrow t$ CAP $T \mid b^{k n} t$ CAP $T \mid b^{n m} t$ RIGHT $T \mid b^{r n}$

$\mathbf{L E F T} \rightarrow t \mathbf{C A P} T \mid B^{k n} t$ CAP $T \mid B^{n m} t$ LEFT $T \mid B^{r n}$

for $h \in\{1,2,3, m n-1\}, \quad j \in\{2,3, \ldots, m+1\}, \quad k \in\{1,2,3, \ldots, m-1\}$, $r \in\{1,2,3, \ldots, m\}, \quad$ and $i \in\{1,2,3, \ldots, n-1\}$ 
Grammar for the $\langle b\rangle$ subgroup of $\operatorname{BS}(n, 2 n m)$, where $n=2 k, k>1, m>2$

Start $\rightarrow \epsilon\left|b^{i}\right| B^{i} \mid t$ CAP $T \mid b^{h} t$ CAP $T \mid B^{h} t$ CAP $T$

Start $\rightarrow b^{n m-k} t$ FRONT $T \mid B^{n m-k} t$ BACK $T$

$\mathbf{S t a r t} \rightarrow b^{n m} t \mathbf{C A P}_{\mathbf{R}} T \mid B^{n m} t \mathbf{C A P}_{\mathbf{L}} T$

Start $\rightarrow b^{m n-L} t$ RIGHT $_{\mathbf{1}} T \mid B^{n m-L} t \mathbf{L E F T}_{\mathbf{1}} T$

Start $\rightarrow B^{n m+L} t$ LEFT $T \mid b^{m n+L} t$ RIGHT $T$

$\mathbf{C O N J} \rightarrow t \mathbf{C A P} T\left|b^{j n} t \mathbf{C A P} T\right| B^{j n} t \mathbf{C A P} T \mid b^{n m} t \mathbf{C A P}_{\mathbf{R}} T$

$\mathbf{C O N J} \rightarrow B^{n m} t \mathbf{C A P}_{\mathbf{L}} T$

$\mathbf{C A P} \rightarrow \mathbf{C O N J}\left|b^{n}\right| B^{n}\left|b^{j n+n}\right| B^{j n+n}$

$\mathbf{F R O N T} \rightarrow \mathbf{C O N J}\left|b^{n}\right| b^{j n+n} \mid B^{j n+n}$

$\mathbf{B A C K} \rightarrow \mathbf{C O N J}\left|B^{n}\right| b^{j n+n} \mid B^{j n+n}$

$\mathbf{C A P}_{\mathbf{R}} \rightarrow t \mathbf{C A P} T\left|b^{J n} t \mathbf{C A P} T\right| b^{n(m-1)} t$ FRONT $T \mid b^{H n}$

$\mathbf{C A P}_{\mathbf{L}} \rightarrow t \mathbf{C A P} T\left|B^{J n} t \mathbf{C A P} T\right| B^{n(m-1)} t \mathbf{B A C K} T \mid B^{H n}$

$\mathbf{R I G H T} \rightarrow t \mathbf{C A P} T\left|b^{J n} t \mathbf{C A P} T\right| b^{n(m-1)} t \mathbf{R I G H T}_{\mathbf{1}} T \mid b^{j n}$

$\mathbf{L E F T} \rightarrow t$ CAP $T \mid B^{J n} t$ CAP $T\left|B^{n(m-1)} t \mathbf{L E F T}_{\mathbf{1}} T\right| B^{j n}$

$\mathbf{R I G H T}_{\mathbf{1}} \rightarrow t \mathbf{C A P} T \mid b^{j n} t$ CAP $T \mid b^{n m} t$ RIGHT $T \mid b^{H n}$

$\mathbf{L E F T}_{\mathbf{1}} \rightarrow t \mathbf{C A P} T\left|B^{j n} t \mathbf{C A P} T\right| B^{n m} t \mathbf{L E F T} T \mid B^{H n}$

for $J \in\{1,2,3, \ldots, m-2\}$,

$j \in\{1,2,3, \ldots, m-1\}$,

$h \in\{1,2,3, \ldots, m n-k-1\}$,

$H \in\{1,2,3, \ldots, m\}$,

$L \in\{1,2,3, \ldots, k-1\}$,

$i \in\{1,2,3, \ldots, n m+k\}$ 
Grammar for the $\langle b\rangle$ subgroup of $\operatorname{BS}(n, 2 n m)$, assuming $n=2 k+1$ and $k>1$

Start $\rightarrow \epsilon\left|b^{i}\right| B^{i}|t \mathbf{C A P} T| b^{h} t \mathbf{C A P} T \mid B^{h} t \mathbf{C A P} T$

Start $\rightarrow b^{n m-k-1} t$ FRONT $T \mid B^{n m-k-1} t$ BACK $T$

Start $\rightarrow b^{n m-k} t \mathbf{R I G H T}_{\mathbf{3}} T \mid B^{n m-k} t \mathbf{L E F T}_{\mathbf{3}} T$

Start $\rightarrow B^{n m-L} t \mathbf{L E F T}_{\mathbf{1}} T \mid b^{m n-L} t$ RIGHT $_{\mathbf{1}} T$

Start $\rightarrow B^{n m} t \mathbf{C A P}_{\mathbf{L}} T \mid b^{n m} t \mathbf{C A P}_{\mathbf{R}} T$

Start $\rightarrow B^{n m+L} t \mathbf{L E F T}_{\mathbf{2}} T \mid b^{m n+L} t$ RIGHT $_{\mathbf{2}} T$

Start $\rightarrow b^{n m+k} t$ RIGHT $_{\mathbf{6}} T \mid B^{n m+k} t$ LEFT $_{\mathbf{6}} T$

$\mathbf{C O N J} \rightarrow t \mathbf{C A P} T\left|b^{j n} t \mathbf{C A P} T\right| B^{j n} t \mathbf{C A P} T \mid b^{n m} t \mathbf{C A P}_{\mathbf{R}} T$

$\mathbf{C O N J} \rightarrow B^{n m} t \mathbf{C A P}_{\mathbf{L}} T$

$\mathbf{C A P} \rightarrow \mathbf{C O N J}\left|b^{n}\right| B^{n}\left|b^{j n+n}\right| B^{j n+n}$

$\mathbf{F R O N T} \rightarrow \mathbf{C O N J}\left|b^{n}\right| b^{j n+n} \mid B^{j n+n}$

$\mathbf{B A C K} \rightarrow \mathbf{C O N J}\left|B^{n}\right| b^{j n+n} \mid B^{j n+n}$

$\mathbf{C A P}_{\mathbf{R}} \rightarrow t \mathbf{C A P} T\left|b^{J n} t \mathbf{C A P} T\right| b^{n(m-1)} t$ FRONT $T \mid b^{H n}$

$\mathbf{C A P}_{\mathbf{L}} \rightarrow t \mathbf{C A P} T\left|B^{J n} t \mathbf{C A P} T\right| B^{n(m-1)} t \mathbf{B A C K} T \mid b^{H n}$

$\mathbf{R I G H T}_{\mathbf{1}} \rightarrow t \mathbf{C A P} T \mid b^{j n} t$ CAP $T\left|b^{n m} t \mathbf{R I G H T}_{\mathbf{2}} T\right| b^{H n}$

$\mathbf{R I G H T}_{\mathbf{2}} \rightarrow t \mathbf{C A P} T\left|b^{J n} t \mathbf{C A P} T\right| b^{n(m-1)} t \mathbf{R I G H T}_{\mathbf{1}} T \mid b^{j n}$

$\mathbf{R I G H T}_{\mathbf{3}} \rightarrow t \mathbf{C A P} T \mid b^{j n} t$ CAP $T\left|b^{n m} t \mathbf{R I G H T}_{\mathbf{4}} T\right| b^{H n}$

$\mathbf{R I G H T}_{\mathbf{4}} \rightarrow t \mathbf{C A P} T\left|b^{J n} t \mathbf{C A P} T\right| b^{n(m-1)} t \mathbf{R I G H T}_{\mathbf{3}} T \mid b^{H n}$

RIGHT $_{\mathbf{5}} \rightarrow t$ CAP $T \mid b^{J n} t$ CAP $T \mid b^{n(m-1)} t$ FRONT $T$

RIGHT $_{\mathbf{5}} \rightarrow b^{n m} t \mathbf{R I G H T}_{\mathbf{6}} T \mid b^{H n}$

RIGHT $_{\mathbf{6}} \rightarrow t$ CAP $T \mid b^{J n} t$ CAP $T \mid b^{n(m-1)} t$ RIGHT $_{\mathbf{5}} T \mid b^{j n}$

$\mathbf{L E F T}_{\mathbf{1}} \rightarrow t \mathbf{C A P} T\left|B^{j n} t \mathbf{C A P} T\right| B^{n m} t \mathbf{L E F T}_{\mathbf{2}} T \mid B^{H n}$

$\mathbf{L E F T}_{\mathbf{2}} \rightarrow t \mathbf{C A P} T\left|B^{J n} t \mathbf{C A P} T\right| B^{n(m-1)} t \mathbf{L E F T}_{\mathbf{1}} T \mid B^{j n}$

$\mathbf{L E F T}_{\mathbf{3}} \rightarrow t \mathbf{C A P} T\left|B^{j n} t \mathbf{C A P} T\right| B^{n m} t \mathbf{L E F T}_{\mathbf{4}} T \mid B^{H n}$

$\mathbf{L E F T}_{\mathbf{4}} \rightarrow t \mathbf{C A P} T\left|B^{J n} t \mathbf{C A P} T\right| B^{n(m-1)} t \mathbf{L E F T}_{\mathbf{3}} T \mid B^{H n}$

$\mathbf{L E F T}_{\mathbf{5}} \rightarrow t \mathbf{C A P} T\left|B^{J n} t \mathbf{C A P} T\right| B^{n(m-1)} t$ BACK $T$

$\mathbf{L E F T}_{\mathbf{5}} \rightarrow B^{n m} t \mathbf{L E F T}_{\mathbf{6}} T \mid B^{H n}$

$\mathbf{L E F T}_{\mathbf{6}} \rightarrow t \mathbf{C A P} T\left|B^{J n} t \mathbf{C A P} T\right| B^{n(m-1)} t \mathbf{L E F T}_{\mathbf{5}} T \mid B^{j n}$

for $j \in\{1,2,3, \ldots, m-1\}, \quad h \in\{1,2,3, \ldots, n m-k-2\}, \quad J \in\{1,2,3, \ldots, m-2\}$,

$H \in\{1,2,3, \ldots, m\}, \quad L \in\{1,2,3, \ldots, k-1\}, \quad$ and $\quad i \in\{1,2,3, \ldots, n m+k+1\}$

\section{References}

1. J. Adams and E. M. Freden, 'A context-sensitive combing associated with Baumslag-Solitar 2, 7', Preprint.

2. G. Baumslag and D. Solitar, 'Some two-generator one-relator non-Hopfian groups', Bull. Amer. Math. Soc. 68 (1962) 199-201.

3. M. BraziL, 'Growth functions for some nonautomatic Baumslag-Solitar groups', Trans. Amer. Math. Soc. 342 (1994) $137-154$. 
4. G. Buntrock and K. Loryś, On growing context-sensitive languages, Lecture Notes in Computer Science, 623 (Springer, Berlin, 1992) 77-88.

5. J. W. CANnon, 'The combinatorial structure of cocompact discrete hyperbolic groups', Geom. Dedicata 16 (1984) 123-148.

6. D. J. Collins, M. Edjvet and C. P. Gill, 'Growth series for the group $\left\langle x, y \mid x^{-1} y x=y^{l}\right\rangle$ ', Arch. Math. (Basel) 62 (1994) no. 1, 1-11.

7. P. DE LA HARPE, Topics in geometric group theory (The University of Chicago Press, Chicago, IL, 2000).

8. V. DiekerT and J. LAun, 'On computing geodesics in Baumslag-Solitar groups', arXiv:0907.5114v2 [math.GR].

9. M. EDJvet and D. L. Johnson, 'The growth of certain amalgamated free products and HNN extensions', J. Aust. Math. Soc. Ser. A 52 (1992) no. 3, 285-298.

10. M. EldER, 'A linear-time algorithm to compute geodesics in solvable Baumslag-Solitar groups', arXiv:0903.0216v3 [math.GR].

11. D. B. A. Epstein, J. W. Cannon, D. F. Holt, S. V. F. Levy, M. S. Paterson and W. P. Thurston, Word processing in groups (Jones-Bartlett, Boston, MA, 1992).

12. B. FArB and L. Moser, 'A rigidity theorem for the solvable Baumslag-Solitar groups', Invent. Math. 131 (1998) 419-451.

13. P. Flajolet and R. Sedgewick, Analytic combinatorics (Cambridge University Press, Cambridge, 2008).

14. E. M. Freden, J. Adams and J. Schofield, 'Growth in Baumslag-Solitar groups II: asymptotics', in preparation.

15. E. M. Freden and J. Schofield, 'The growth of Higman 3', J. Group Theory 11 (2008) 227-298.

16. R. H. Gilman, 'A shrinking lemma for indexed languages', Theoret. Comput. Sci. 163 (1996) 277-281.

17. J. E. Hopcroft and J. D. Ullman, Introduction to automata theory, languages, and computation (Addison-Wesley, Reading, MA, 1979).

18. Source code for some growth computations in Baumslag-Solitar groups, http://antares.cciet.suu.edu/papers.html.

19. K. ViJaY-Shanker, 'A study of tree adjoining grammars', PhD Thesis, University of Pennsylvania, 1987.

20. K. ViJAY-Shanker and D. J. Weir, 'The equivalence of four extensions of context-free grammars', Math. Syst. Theory 27 (1994) 511-546.

Eric M. Freden

Department of Mathematics

Southern Utah University

Cedar City UT

USA

freden@suu.edu

Jennifer Schofield

Department of Mathematics

Brigham Young University

Provo UT

USA

jens@math.byu.edu
Teresa Knudson

Mohave Community College

North Mohave Campus

Colorado City AZ

USA

tcawley@mohave.edu 